\title{
Thermal Metasurfaces: Complete Emission Control by Combining Local and Nonlocal Light-Matter Interactions
}

\author{
Adam C. Overvig $\odot,{ }^{1}$ Sander A. Mann $\odot,{ }^{1}$ and Andrea Alù $\oplus^{1,2, *}$ \\ ${ }^{1}$ Photonics Initiative, Advanced Science Research Center, City University of New York, \\ New York, New York 10031, USA \\ ${ }^{2}$ Physics Program, Graduate Center of the City University of New York, New York, New York 10016, USA
}

(Received 31 December 2020; revised 11 March 2021; accepted 30 April 2021; published 4 June 2021)

\begin{abstract}
Metasurfaces have been enabling the miniaturization and integration of complex optical functionalities within an ultrathin platform by engineering the scattering features of localized modes. However, these efforts have mostly been limited to the manipulation of externally produced coherent light, e.g., from a laser. In parallel, the past two decades have seen the development of structured surfaces that emit partially coherent radiation via thermally populated, spatially extended (nonlocal) modes. However, the control over thermally emitted light is severely limited compared to optical metasurfaces, and even basic functionalities such as unidirectional emission to an arbitrary angle and polarization remain elusive. Here, we derive the necessary conditions to achieve full control over thermally emitted light, pointing to the need for simultaneously tailoring local and nonlocal scattering features across the structure. Based on these findings, we introduce a platform for thermal metasurfaces based on quasibound states in the continuum that satisfies these requirements and completes the program of compactification of optical systems by enabling a full degree of control of partially coherent light emission from structured thin films, including unidirectional emission of circularly polarized light, focusing, and control of spatial and temporal coherence, as well as wave-front control with designer spin and angular orbital momenta.
\end{abstract}

DOI: 10.1103/PhysRevX.11.021050

Subject Areas: Metamaterials, Optics

\section{INTRODUCTION}

Thermal emission is arguably the most established technique for producing light at optical frequencies, and its use remains ubiquitous today. From the time of Newton, manipulation of light emitted by a blackbody proceeds by placing a series of optical elements in the optical pathway to control its spectrum, coherence properties, polarization state, and spatial profile (wave-front shape). While bulky, such setups are capable of a large degree of control over light produced by a heated element. For instance, Fig. 1(a) schematically depicts such a "bulk optics" setup aimed at producing narrow-band light with designer spin angular momentum (SAM) and orbital angular momentum (OAM), wherein emission from a blackbody is spatially filtered by a pinhole, collimated by a lens, filtered by a monochromator, polarized by a linear polarizer, converted to circular

\footnotetext{
* Corresponding author. aalu@gc.cuny.edu

Published by the American Physical Society under the terms of the Creative Commons Attribution 4.0 International license. Further distribution of this work must maintain attribution to the author(s) and the published article's title, journal citation, and DOI.
}

polarization by a quarter wave plate, and finally passed through a phase plate imparting the desired OAM profile.

In this framework, a basic requirement is the production of wave fronts with sufficient coherence such that light may be collimated with the desired spatial and polarization properties imparted to it. Spatial coherence naturally increases upon propagation [1-3] or through spatial filtering, implying that an incoherent source can acquire coherence at the cost of large volumes and inefficiencies. A lens is then able to collimate this spatially coherent wave into a narrow momentum range. Likewise, a monochromator can spectrally filter the signal in order to produce a wave with the desired narrow range of frequencies. Since the temporal coherence is inversely related to the bandwidth $[2,4,5]$, these first steps of the bulk optics approach convert incoherent light into partially coherent light at the cost of rejecting much of the optical energy.

In the past decade, substantial progress has been made in the context of compactifying the latter stages of the system shown in Fig. 1(a) by the use of structured optical materials, or metamaterials. Particularly, there has been significant focus in metamaterials and structured thin films (metasurfaces) aimed at manipulating externally produced coherent light (e.g., light from a laser) or light with a large degree of coherence (e.g., light that is prepared as in the beginning stages of the bulk optics setup). These metasurfaces have 


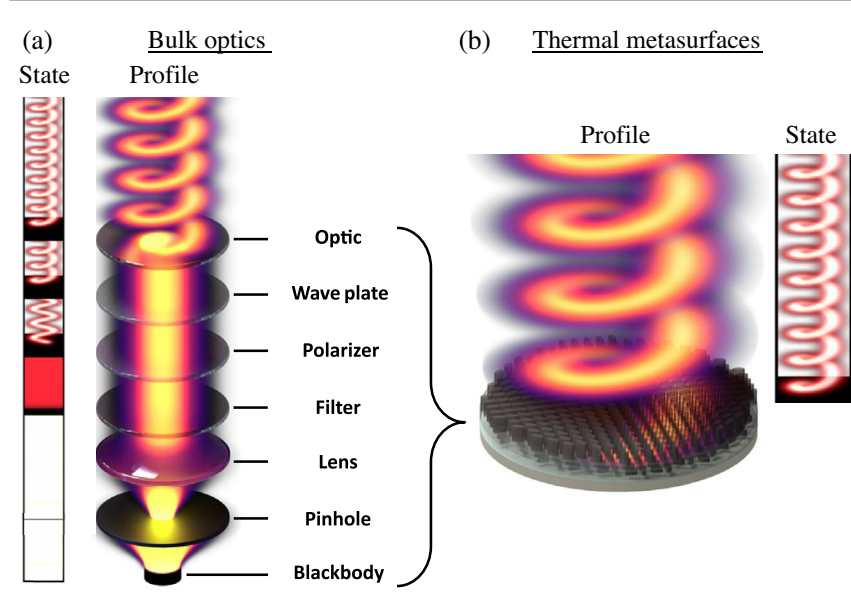

FIG. 1. Comparison of conventional optics and thermal metasurfaces producing light with desired SAM and OAM. (a) In the bulk optics approach, the emission from a blackbody is spatially filtered, collimated, spectrally filtered, polarized, retarded, and shaped by separate components, leading to large inefficiencies and bulky setups. (b) With a thermal metasurface, thermal emission is shaped at the source by a subwavelength film.

been establishing an ultrathin platform wherein designer polarization and spatial profiles are imparted to light within a subwavelength structured film $[6,7]$. Recent demonstrations [8] show complete control over phase and polarization with subwavelength spatial resolution [9,10], amplitude, and phase [11,12] and even simultaneous control of amplitude, phase, and polarization at a single wavelength $[13,14]$. Essentially, these devices act on the incoming wave front by locally manipulating the impinging wave front through their subwavelength units; nearest-neighbor interactions among these elements are often ignored so that reference to a library of precomputed optical responses may guide the configuration of a device geometry with the desired functionality. Because they operate based on independent scatterers arrayed across the surface, they are referred to as spatially "local" metasurfaces.

While this progress has enabled exciting opportunities for compact manipulation of external, coherent sources, incorporating all stages of the system in Fig. 1(a) into a single compact structure is still an outstanding challenge despite the enticing benefits regarding the size, weight, and power of custom optical sources. The difficulties involved in constructing such a structure, which we call a "thermal metasurface" [Fig. 1(b)], are a direct result of the inherent incoherence involved with the fluctuations responsible for thermal emission, which is incompatible with the large spatial and temporal coherence required for conventional metasurfaces. In other words, conventional metasurfaces excited by a coherent source work well because each element is driven with a well-defined phase, enabling effective control over the transmitted or reflected wave front. In thermal metasurfaces, on the other hand, light emanates from the metasurface elements themselves through spontaneous processes, and no coherence exists between the processes in different elements. As a result, combining all steps in Fig. 1(a) into one, i.e., manipulating the incoherent thermal processes to generate a partially coherent, complex wave front, is significantly more challenging than designing an externally and coherently driven metasurface with complex functionalities.

Despite these challenges, the past two decades have seen some progress in this context [15]. In 2002, Greffet et al. seminally demonstrated that a patterned silicon carbide surface can be designed to emit thermal light with enhanced coherence compared to an unpatterned interface [16]. This surprising phenomenon, wherein random thermal fluctuations produce correlated electromagnetic fields in the far field, is made possible by the near-field details: Thermally populated surface waves propagate laterally before scattering to the far field, correlating the emission across distances greater than the wavelength of light. Since then, the capabilities rooted in this nonlocal phenomenon have been extended, developing a platform for compactification of a few of the first stages in Fig. 1(a). For example, some degree of control over the emission spectrum of a given material is demonstrated through the use of extended modes supported by waveguides [17] and photonic crystals [18-22], surface phonon polaritons [23-25], and with arrays of resonant elements tailored in space [26-28]. Generally speaking, in these approaches, spatial coherence is provided to the emitted beam by using gratings to couple to surface waves or other nonlocal modes (modes supported by many adjacent structural elements) [16,29-31] or with multilayer structures [32,33], while tailoring the bandwidth of emission (which is relatively narrow in these structures) controls the temporal coherence of thermal radiation [16,34]. These structures can also provide selectivity for a desired polarization: For example, surface waves are typically transverse magnetic (TM) polarized, and, therefore, these devices preferentially emit TM-polarized thermal radiation. In this way, the first three steps of the bulk optics device in Fig. 1(a) may be collapsed into a thin device to a certain extent, with great potential for substantially reducing the size, weight, power, and complexity of the entire optical system.

Given the recent expansion of scope of the term metasurface to capture a broad class of optically thin structured materials composed of subwavelength features, it may be argued that these results from the past two decades may fall under the broad class of thermal metasurfaces. Yet, metasurfaces are more accurately associated with an advanced degree of control over the wave front of light enabled by the careful patterning of spatially localized modes across the surface-such control is notably lacking in the control over thermal light. In other words, despite this progress, control over the polarization state and directionality of thermally emitted light remains so far limited. For example, thermal generation of circularly polarized light with $28 \%$ efficiency 
is demonstrated by integrating the bulk optics stack into a multilayer structure separated by spacers, where different layers impart specific operations to the emitted beam [35]. In terms of single, compact elements manipulating thermal emission, there have recently been a few demonstrations of more elaborate functionalities, such as circularly polarized $(C P)$ [36-40] or unidirectional emission [41] and even partial focusing [42]. Notably, mesoscopic structures with carefully engineered coupling have been recently demonstrated to enable focused and holographic emission [43]. However, a comprehensive framework in the mold of optical metasurfaces (i.e., based on rational design of subwavelength structures patterning the overall wave front) enabling thermal emission of arbitrary polarization states into any specific direction, and, more generally, control over the degree of spatial and temporal coherence and over the spatial wave front, has not yet been developed. For instance, even a thermal emitter exhibiting a combination of the previously demonstrated achievements simultaneously (i.e., unidirectional asymmetric emission of $C P$ light) has not been demonstrated, let alone arbitrary wave-front generation. This endeavor has enormous appeal beyond the previously stated footprint advantages: For example, a thermal emitter that emits power only at a desired frequency, into the desired optical mode, is inherently much more efficient than a system where undesired modes and frequencies must be filtered out [for instance, the pinhole, polarizer, and spectral filter in Fig. 1(a) all reduce the overall efficiency].

Relatedly, there exist similar long-standing challenges in efficiently extracting and collimating emission from lightemitting diodes (LEDs) [44,45]. Since luminescence is similar to thermal emission, but with a nonzero chemical potential [46], a comprehensive framework for efficiently generating complex wave fronts from incoherent oscillations in thin planarized structures is also applicable to luminescence. Similar to the case of thermal radiation, luminescence of circularly polarized light has been demonstrated using chiral structures [47]. Unidirectional emission from a luminescent structure has also recently been demonstrated [48], but complete control over the direction, polarization state, and wave front of luminescence emanating from a metasurface remains equally elusive. For instance, chiral emission remains limited to bidirectional emission (wherein the directional emission of one spin is balanced by emission of the opposite spin in the opposite direction), as seen in Ref. [49]. However, by using a spatially localized source that couples energy to a unidirectionally traveling surface mode, the bidirectional symmetry may be broken and complex wave fronts may emerge. Vector vortex beams have been demonstrated by focused optical excitation of luminescence materials at the center of a spiral plasmonic device [50,51], albeit only for TM polarization. Unfortunately, such approaches achieve their functionality by making the spatial incoherence of the luminescence irrelevant via the tight focusing, intrinsically coming at the cost of limiting the efficiency, throughput, and scalability, akin to the advantages and drawbacks of the pinhole in Fig. 1(a). Finally, in the context of enhancing luminescence, devices based on quasibound states in the continuum (QBICs) demonstrate substantially narrowed emission spectra via long-lived states controlled by a symmetry-lowering perturbation, implying maximized efficiency of emission into a quasimonochromatic beam with the desired wavelength [52].

In this paper, we introduce a general framework for thermal metasurfaces that enables ultrathin thermal sources to directly emit custom wave fronts [Fig. 1(b)]. Our framework demonstrates that, to do so, lessons must be learned from both "local" metasurfaces [6-14], wherein each metasurface element affects only the local phase and amplitude of the impinging wave front, and "nonlocal" metasurfaces [53-57], which function via spatially extended optical modes such as surface waves, in-plane guided waves, or QBICs [58]. The recent insights provided by nonlocal metasurfaces naturally connect to thermal metasurfaces, because in both cases engineering of longrange interactions (i.e., correlations across many adjacent elements) is required. However, in the following, we show that, while nonlocality is necessary for adding coherence to the thermal emission within the desired flat form factor, it is not sufficient for arbitrary control in reciprocal systems satisfying time-reversal symmetry: Simultaneous control of both the local and nonlocal properties is required. Such a feat requires resolution of an inherent tension between local and nonlocal phenomena: Locality entails rapidly and widely varying subwavelength structures that shape the wave front, while nonlocality entails slowly varying features that support extended modes with well-controlled properties. We solve this challenge by exploiting the symmetry protection offered by nonlocal QBIC modes. We design structures wherein the nonlocal mode is introduced upon application of small geometric perturbations to the local pattern. By the judicious use of selection rules governing QBICs [54], we selectively break only symmetries that are otherwise preserved by the local pattern, thereby offering a rational design framework wherein local and nonlocal effects are separable by symmetry. Within this hybrid local-nonlocal metasurface platform, all stages in Fig. 1(a) are integrated into a single ultrathin device, maximizing conversion efficiency of heat (in the case of thermal radiation) or chemical potential (in the case of luminescence) into light with the desired polarization state and direction and largely minimizing the overall footprint of the optical system. With no external optics necessary, thermal metasurfaces represent a completion of the program of compactification of bulk optics long pursued by the metasurface community.

The paper is structured as follows. First, in Sec. II, we identify the key parameters of the optical wave front that a 
thermal metasurface must control. We then introduce in Sec. III a dual perturbative framework for controlling the emissivity and temporal coherence of light by utilizing the band-edge mode associated with quasibound states in the continuum controlled by suitably perturbed dielectric structures. We show that the band structure and $Q$ factor together can control to a large extent the spatial coherence of emitted light. Up to this point, our results offer a generalized platform for the first half of the compactification program pursued by nonlocal metasurfaces, enabling control of emissivity and temporal and spatial coherence of quasimonochromatic light. To incorporate the remaining functionalities required for a complete compactification, Sec. IV outlines basic constraints that thermal metasurfaces are bound to, on the basis of time-reversal invariance and reciprocity. We find that a thermal metasurface may not shape light by purely controlling the nonlocal response, i.e., by only engineering the QBIC, but instead it requires simultaneous control over its local and nonlocal responses. Based on this result, Sec. V demonstrates thermal metasurfaces composed of two-layer planarized structures made of doped silicon placed above a mirror plane, enabling arbitrary control over the emitted polarization state, amplitude, and bandwidth. By spatially grading a geometric phase inherited from the degeneracy of the longitude of the poles of the Poincaré sphere, we also demonstrate unidirectional thermal emission with selected optical spin and next extend this demonstration to aperiodic wave-front shaping by showing focused thermal emission. Finally, we demonstrate that azimuthally wrapping the introduced geometric phase enables the design of thermal metasurfaces generating quasimonochromatic wave fronts with designer spin and orbital angular momenta. Overall, our results provide a framework for planarized ultrathin thermal emitters with arbitrary control over amplitude, polarization, local phase, and coherence of the generated quasimonochromatic light.

\section{BACKGROUND: PARTIALLY COHERENT WAVEFRONT PARAMETERIZATION}

To shape our discussion and clarify what a thermal metasurface can, in principle, be capable of, we first define the parameters characterizing its generated wave front. For this purpose, we compare the coherent wave fronts engineered by conventional metasurfaces to the partially coherent wave fronts generated by thermal metasurfaces. As depicted in Fig. 2(a), conventional metasurfaces are excited by an external coherent source (e.g., from a laser), locally scattering the impinging light at the metasurface plane $(z=0)$ in order to control the optical wave front at a parallel plane of interest $(z=d)$. Guided by the HuygensFresnel principle, a coherent metasurface locally tailors the amplitude $A$, phase $\Phi$, and polarization response, characterized by the ellipticity parameters $\psi$ and $\chi$. This tailoring is typically achieved using a precomputed library of unit cell geometries, where nearest-neighbor interactions are ignored, implying a strictly local response. For a scalar wave front, i.e., $\psi$ and $\chi$ fixed across the device, the field along the metasurface $E(\boldsymbol{\rho}, z=0)$ produces a field $E(\boldsymbol{\rho}, z=d)$ at a parallel plane according to [1]

$E(\boldsymbol{\rho}, z=d)=\int d \boldsymbol{\rho}^{\prime} E\left(\boldsymbol{\rho}^{\prime}, z=0\right) \exp \left(i k_{0}\left|\boldsymbol{\rho}-\boldsymbol{\rho}^{\prime}\right|\right) /\left|\boldsymbol{\rho}-\boldsymbol{\rho}^{\prime}\right|$,

where we leave the frequency dependence implicit and we use the spatial coordinates $\mathbf{r}=(\boldsymbol{\rho}, z)$ with $\boldsymbol{\rho}=(x, y)$. (a)

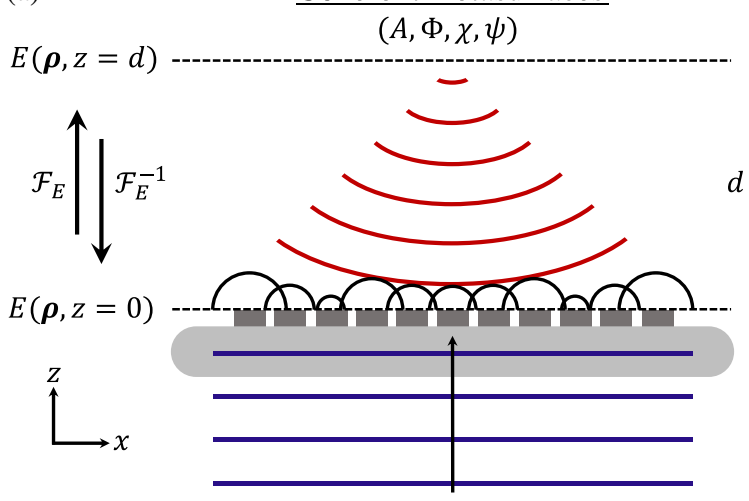

Laser excitation (b) Thermal metasurfaces

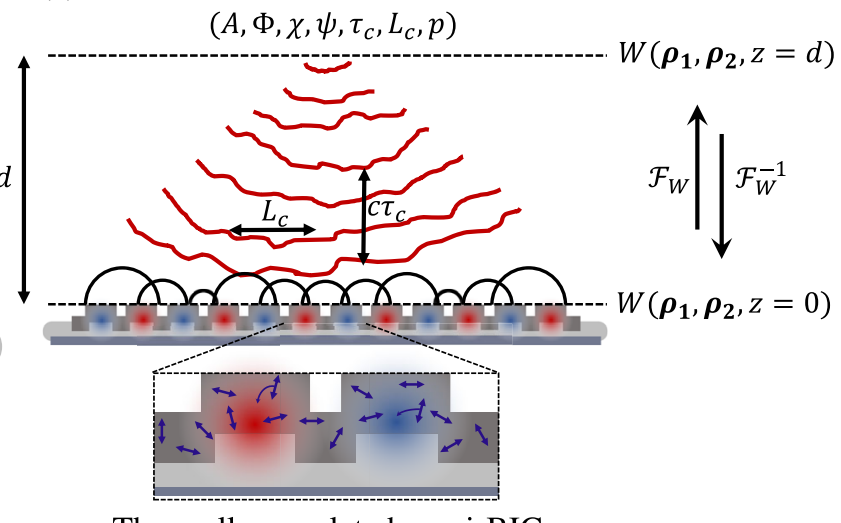

Thermally populated quasi-BIC

FIG. 2. Comparison of coherent and thermal metasurfaces. (a) A coherent metasurface transforms an incident waveform (blue) into the desired waveform (red) by spatially varying the scattering along the surface, visualized as Huygens' wavelets (black). Each wavelet is characterized by a combination of amplitude, phase, and polarization state, which in concert produce the desired far-field profile. The coherence of the system implies that the mapping from metasurface to far field is invertible. (b) A thermal metasurface composed of a patterned structure over a ground plane supporting a thermally populated global mode (blue) whose out-of-plane scattering is locally tailored to produce a partially coherent wave form of choice. 
Ignoring the nonradiative (near-field) field components, the relationship between $E(\boldsymbol{\rho}, z=0)$ and $E(\boldsymbol{\rho}, z=d)$ is invertible as

$E(\boldsymbol{\rho}, z=0)=\int d \boldsymbol{\rho}^{\prime} E\left(\boldsymbol{\rho}^{\prime}, z=d\right) \exp \left(-i k_{0}\left|\boldsymbol{\rho}-\boldsymbol{\rho}^{\prime}\right|\right) /\left|\boldsymbol{\rho}-\boldsymbol{\rho}^{\prime}\right|$,

implying that we may specify the output field of the metasurface required to generate the desired field in the far field. This mapping of electric fields is schematically sketched in Fig. 2(a) by the side arrows and denoted with $\mathcal{F}_{E}$ for the forward mapping and with $\mathcal{F}_{E}^{-1}$ for the inverted mapping, consistent with the foundations of holographic techniques [59]. For these local metasurfaces, the field exiting the metasurface is simply given by

$$
E(\boldsymbol{\rho}, z=0)=\tau(\boldsymbol{\rho}) E_{\text {in }}(\boldsymbol{\rho}, z=0),
$$

which, together with Eq. (2), specifies the required local amplitude and phase response of the metasurface $\tau(\boldsymbol{\rho})$. This description can be straightforwardly extended to a vectorial form, enabling complete point-by-point control of the outgoing values of $A, \Phi, \psi$, and $\chi$.

In contrast, a thermal metasurface produces a partially coherent wave front parameterized by more degrees of freedom than a coherent one. The wave front is generated indirectly, mediated by a thermally populated QBIC that correlates optical energy across the surface before scattering to the far field [Fig. 2(b)]. In general, the wave front emanating from the metasurface is characterized not only by $A, \Phi, \psi$, and $\chi$, but also by its temporal coherence $\tau_{c}$, spatial coherence length $L_{c}$, and degree of polarization $p$. Notably, the phase is only locally physical: Partially coherent wave fronts are statistically described by ensemble averages that destroy the phase information over sufficiently large time periods and distances. Within the coherence volume of the wave, however, the phase is necessary to describe the optical wave front; e.g., the local phase front still describes the direction of the optical energy flow. In particular, for scalar wave fronts, a partially coherent wave is described by the cross-spectral density [2]

$$
W\left(\boldsymbol{\rho}_{1}, \boldsymbol{\rho}_{2}, z\right)=\left\langle E^{*}\left(\boldsymbol{\rho}_{1}, z\right) E\left(\boldsymbol{\rho}_{2}, z\right)\right\rangle,
$$

where the angle brackets denote an appropriate time average or ensemble average, which is characterized by the coherence properties of the wave front. The cross-spectral density at the metasurface plane $W\left(\boldsymbol{\rho}_{1}, \boldsymbol{\rho}_{2}, z=0\right)$ produces a field $W\left(\boldsymbol{\rho}_{1}, \boldsymbol{\rho}_{2}, z=d\right)$ at a parallel plane according to

$$
\begin{aligned}
& W\left(\boldsymbol{\rho}_{1}, \boldsymbol{\rho}_{2}, d\right) \\
& \quad=\iint d \boldsymbol{\rho}_{1}^{\prime} d \boldsymbol{\rho}_{2}^{\prime} G^{*}\left(\boldsymbol{\rho}_{1}-\boldsymbol{\rho}_{1}^{\prime}, d\right) G\left(\boldsymbol{\rho}_{2}-\boldsymbol{\rho}_{2}^{\prime}, d\right) W\left(\boldsymbol{\rho}_{1}^{\prime}, \boldsymbol{\rho}_{1}^{\prime}, 0\right),
\end{aligned}
$$

where

$$
G\left(\boldsymbol{\rho}-\boldsymbol{\rho}^{\prime}, d\right)=\exp \left(i \frac{\omega}{c} \sqrt{\left|\boldsymbol{\rho}-\boldsymbol{\rho}^{\prime}\right|^{2}+d^{2}}\right) / \sqrt{\left|\boldsymbol{\rho}-\boldsymbol{\rho}^{\prime}\right|^{2}+d^{2}} .
$$

Again ignoring the near-field components, the relationship between $W\left(\boldsymbol{\rho}_{1}, \boldsymbol{\rho}_{2}, 0\right)$ and $W\left(\boldsymbol{\rho}_{1}, \boldsymbol{\rho}_{2}, d\right)$ is invertible to

$$
\begin{aligned}
W\left(\boldsymbol{\rho}_{1}, \boldsymbol{\rho}_{2}, 0\right)= & \iint d \boldsymbol{\rho}_{1}{ }_{1} d \boldsymbol{\rho}_{2}{ }_{2} G\left(\boldsymbol{\rho}_{1}-\boldsymbol{\rho}_{1}^{\prime}, d\right) \\
& \times G^{*}\left(\boldsymbol{\rho}_{2}-\boldsymbol{\rho}_{2}{ }_{2}, d\right) W\left(\boldsymbol{\rho}_{1}^{\prime}{ }_{1}, \boldsymbol{\rho}_{2}{ }_{2}, d\right),
\end{aligned}
$$

meaning that one may compute the required output crossspectral density of the thermal metasurface in order to generate a desired far field. This mapping is denoted $\mathcal{F}_{W}$ and the inverted mapping $\mathcal{F}_{W}^{-1}$ in Fig. 2(b), and it may be seen as the extension of holography to partially coherent waves.

A key property of the cross-spectral density is the information that it contains about the field coherence. In the space-frequency domain, this information is encapsulated within the spectral degree of coherence

$$
\mu\left(\mathbf{r}_{1}, \mathbf{r}_{2}, \omega\right)=\frac{W\left(\mathbf{r}_{1}, \mathbf{r}_{2}, \omega\right)}{\sqrt{W\left(\mathbf{r}_{1}, \mathbf{r}_{1}, \omega\right)} \sqrt{W\left(\mathbf{r}_{2}, \mathbf{r}_{2}, \omega\right)}},
$$

which is a complex quantity whose magnitude varies between 0 (completely incoherent) and (completely coherent). Note that here we explicitly add the frequency dependence to emphasize that this quantity is defined in the space-frequency domain. Finally, for vector fields, the cross-spectral density is replaced by the electric crossspectral density

$$
\mathbf{W}=\left[\begin{array}{ll}
W_{x x} & W_{x y} \\
W_{y x} & W_{y y}
\end{array}\right]
$$

where

$$
W_{i j}\left(\boldsymbol{\rho}_{1}, \boldsymbol{\rho}_{2}, \omega\right)=\left\langle E_{i}^{*}\left(\boldsymbol{\rho}_{1}, \omega\right) E_{j}\left(\boldsymbol{\rho}_{2}, \omega\right)\right\rangle,
$$

which contains the information about the degree of polarization [2,3],

$$
p(\mathbf{r}, \omega)=\sqrt{1-\frac{4 \operatorname{Det}[\mathbf{W}(\mathbf{r}, \mathbf{r}, \omega)]}{\{\operatorname{Tr}[\mathbf{W}(\mathbf{r}, \mathbf{r}, \omega)]\}^{2}}} .
$$

In the following, we develop a general framework for thermal metasurfaces aimed at controlling all parameters defining the electric cross-spectral density, $A, \Phi, \psi, \chi, \tau_{c}$, $L_{c}$, and $p$, and we demonstrate key functionalities of this platform with concrete examples. In Sec. III A, we show 
that a perturbative framework can govern both the radiative and nonradiative lifetimes of the metasurface at the operating frequency, controlling both the linewidth and peak emissivity simultaneously. In Sec. III B we show that, since the linewidth is related to the lifetime of the mode, this control amounts to complete command of $A$ and $\tau_{c}$. We further show that the metasurface nonlocality determines the spatial coherence of the emitted wave front $L_{c}$, and it can be tailored by band structure engineering. Then, in Sec. IV, we elucidate fundamental constraints that govern the spatial control of emission from thermal metasurfaces, clarifying that a combination of local and nonlocal responses is required to achieve complete spatial control. In Sec. VA, we show an example implementation of a thermal metasurface that emits light to any arbitrary elliptical polarization state while inheriting the control shown in Sec. III, realizing a platform that controls $A$, $\psi, \chi, \tau_{c}$, and $L_{c}$ simultaneously. Then, Sec. V B shows that, when circular polarization is chosen, the geometric phase enables control of $\Phi$ in place of $\psi$, enabling directional emission of light, emission of focused light, and emission of light with designer SAM and OAM. For simplicity, we constrain ourselves to circular polarization, which affords a convenient method to control the phase through the geometric phase. We stress, however, that the introduced platform is capable of controlling the phase and polarization of emitted light even more generally by suitably tailoring the propagation phase of the local response, as discussed in the Appendix A.

\section{COHERENT THERMAL EMISSION WITH QBICS}

In order to gain control over thermal emission from a structured material mediated by a long lifetime mode, we need command of both its radiative and nonradiative lifetimes. Here, we propose a dual perturbative approach for this purpose: Beginning with a high-symmetry photonic crystal slab in a lossless materials system [Fig. 3(a)], we alter it with both a geometric and a material perturbation (a)

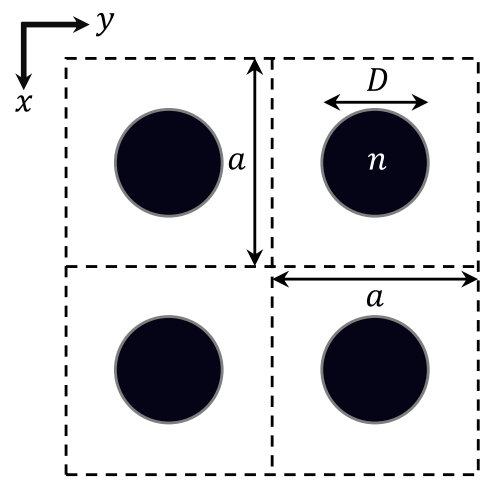

(b)

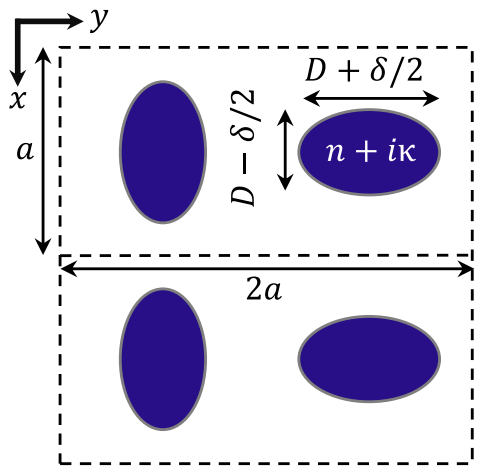

(c)

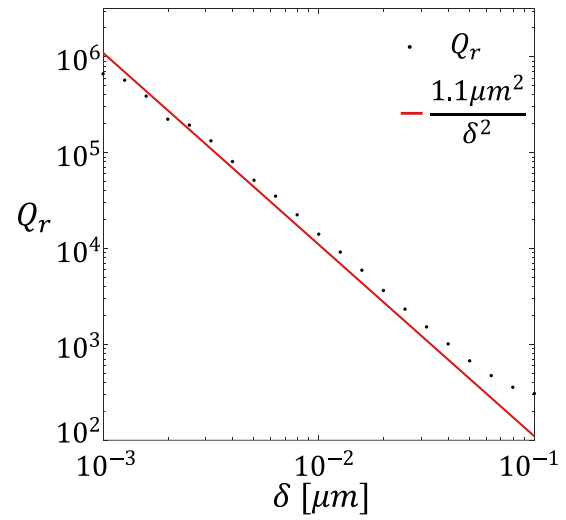

(d)

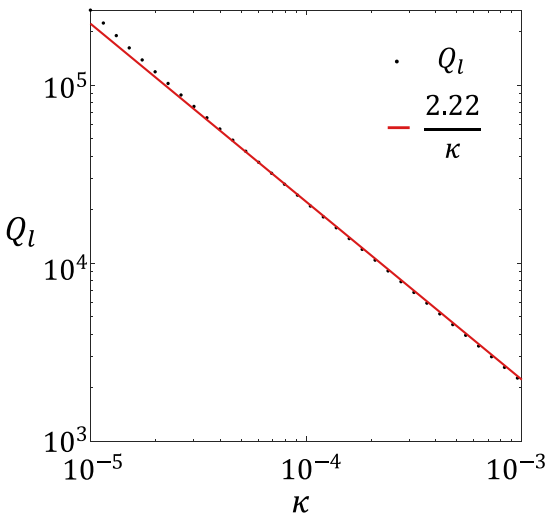

(e)

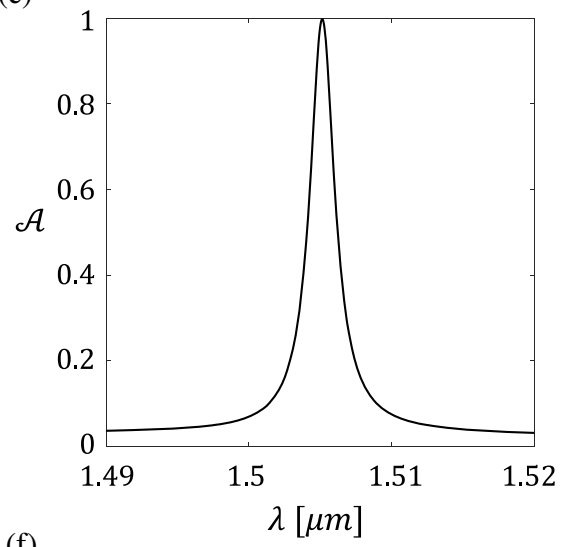

(f)

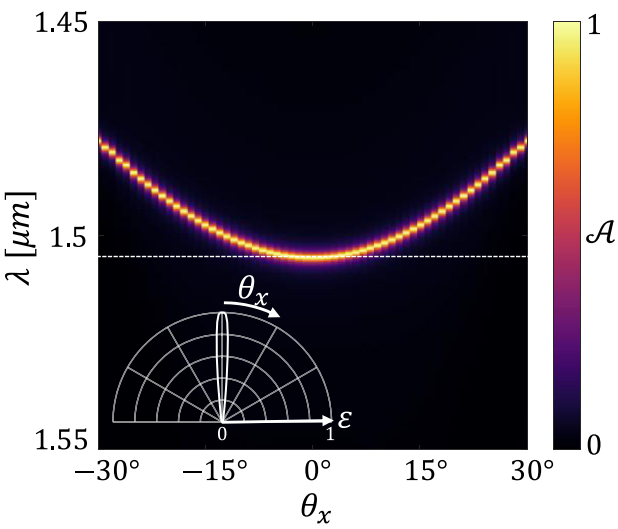

FIG. 3. Perturbative framework for thermally emissive QBICs. (a) An unperturbed high-symmetry lattice of a lossless material with index $n$ is perturbed (b) both geometrically (with asymmetry parameter $\delta$ ) and in index (adding a small extinction coefficient $\kappa$ ). (c) The radiative $Q$ factor (for fixed $k$ ) varies as $Q_{r} \propto 1 / \delta^{2}$, and (d) the nonradiative $Q$ factor (for fixed $\delta$ ) varies as $Q_{l} \propto 1 / \kappa$. Dots in (c,d) are calculated by full-wave simulations, while the red lines show linear fits to these data. (e) Absorption spectrum of the QBIC near the resonant frequency for normal incidence and $x$-polarized light. (f) Absorption spectra for a range of incidence angles, showing perfect absorption following a parabolic angular dispersion. The band-edge mode (dashed white line) emits to a narrow range of angles near the normal (inset). Note: The three-dimensional structure used to calculate (c)-(f) is shown in Figs. 6(a)-6(c) with the values $\theta=\alpha=0$. 
[Fig. 3(b)]. The geometric perturbation, quantified by $\delta$, controls the radiative lifetime of a QBIC supported by the structure, while the material perturbation, quantified by $\kappa$, introduces an imaginary part of the refractive index controlling the nonradiative lifetime. Then, by operating at the band-edge frequency, we ensure that light emits into a small solid angle near normal incidence, with angular spread $\Delta k$. In this way, we can simultaneously tune the lifetime and peak emissivity of a QBIC supported by the thermal metasurface. In the following, we show that, while the lifetime directly controls temporal coherence of the emitted light, the angular spread $\Delta k$ determines and controls its spatial coherence. Numerical results are produced by fullwave simulations using the finite difference time domain method (Lumerical Solutions). The absorption properties of the devices are studied under various illumination conditions, and the emission features are then retrieved due to the relations between absorptivity and emissivity [60].

\section{A. Controlling coherent emission with thermally populated QBICs}

While the approach presented in the following is quite general, for concreteness, we envision the perturbations imparted to our otherwise symmetric structure to be a dimerization of a square lattice of doped silicon nanopillars (refractive index $n=3.45$ when undoped) in air. As shown in Fig. 3(b), the circular pillars are distorted into two ellipses oriented $90^{\circ}$ from one another with major and minor diameters differing by $\delta$, and silicon is altered by the introduction of dopants, perturbing the refractive index of the silicon by a value $\Delta n+i \kappa$. For generality and simplicity, throughout this work, we take these material parameters to be dispersionless, but we note that the monochromaticity may be improved by selecting a dopant with a narrow-band absorption profile, such as erbium dopant atoms implanted into silicon for near-infrared wavelength operation [61]. The dimerization creates and governs the radiative properties of the QBIC $[58,62]$, while the imaginary part of the refractive index allows thermal population of the QBIC in the near field. In particular, the QBIC has a radiative $Q$ factor following $Q_{r}=\omega_{0} / 2 \gamma_{r} \propto$ $1 / \delta^{2}$ [Fig. 3(c)] and a nonradiative $Q$ factor following $Q_{l}=$ $\omega_{0} / 2 \gamma_{i} \propto 1 / \kappa$ [Fig. 3(d)], where $\omega_{0}$ is the resonant frequency, $\gamma_{r}$ is the radiative decay rate of the mode, and $\gamma_{i}$ is the nonradiative decay rate. The dependence of the radiative $Q$ factor on $\delta$ is well established for QBICs [58], and the expression of the nonradiative $Q$ factor as a function of $\kappa$ may be derived based on a perturbative analysis (see Appendix B).

When $\delta$ and $\kappa$ are properly tuned to achieve critical coupling [63,64], $x$-polarized light at normal incidence is perfectly absorbed at the resonant frequency, which produces a Fano line shape [Fig. 3(e)] corresponding to a sharp thermal emission feature. Off normal incidence, similar absorption and emission spectra can be observed, but with a resonant frequency shift following the angular dispersion of the QBIC, shown in Fig. 3(f). Because of reciprocity, this band diagram is necessarily symmetric, implying that at all frequencies are absorbed symmetrically for opposite incidence angles. In other words, for every eigenfrequency $\omega_{i}(k)$, we find a second eigenfrequency $\omega_{i}(-k)=\omega_{i}(k)$. This symmetry in the modal structure appears to suggest that unidirectional emission to an arbitrary angle, without emitting to the reciprocal one, is impossible. However, as we establish in Sec. IV, by tailoring the far-field coupling at $\omega_{i}(k)$ and $\omega_{i}(-k)$, it is possible to realize unidirectional emission at an arbitrary angle in completely reciprocal systems. For now, we notice that at the band edge these two angles converge to a single one at normal incidence, in which case thermal emission occurs unidirectionally along the surface normal [inset in Fig. 3(f)].

Taken as a whole, the results in Fig. 3 imply that our double perturbation allows independent control over radiative and nonradiative lifetimes, enabling the construction of a library of perturbations as we independently vary $\delta$ and $\kappa$ at the basis of the design of thermal metasurfaces. Such a library, along with possible implementations and alternatives, is discussed further in Appendix C, where $Q_{r}$ varies according to the geometric perturbation and $Q_{l}$ according to the dopant concentration. We emphasize that, based on temporal coupled mode theory (TCMT) [64-66], a Fano resonance with radiative $Q$ factor $Q_{r}$ and nonradiative $Q$ factor $Q_{l}$ has an overall $Q$ factor

$$
Q_{0}=\frac{Q_{r} Q_{l}}{Q_{r}+Q_{l}} .
$$

Meanwhile, the absorption $\mathcal{A}$ at resonance follows [63]

$$
\mathcal{A}=\varepsilon=\frac{4 Q_{r} Q_{l}}{\left(Q_{r}+Q_{l}\right)^{2}}
$$

where, by Kirchhoff's law of thermal radiation, we equate it to the thermal emissivity $\varepsilon$. Hence, a thermal metasurface with simultaneously controllable radiative and nonradiative lifetimes has command over its linewidth and peak emissivity. The control shown in Fig. 3, therefore, represents the first step to constructing a metasurface that thermally emits light in a spatially tailorable manner.

\section{B. Temporal and spatial coherence of a thermally populated QBIC}

We are now ready to relate the optical lifetime and angular dispersion near the band edge to the temporal and spatial coherence of light emitted by a thermally populated QBIC. The temporal coherence is determined by its optical lifetime, i.e., its linewidth. The spatial coherence depends on the near field of the thermal source [67-69], in our case corresponding to a characteristic distance over which the QBIC energy propagates laterally in plane before scattering 
out to the far field; it, therefore, also depends on the optical lifetime of the mode. Nevertheless, we find that it additionally depends on the shape of the band structure, and so it may be independently controlled by band structure engineering. In the following, we derive an analytical expression for the spectral degree of coherence based on TCMT, yielding a simple route to control the spatial coherence of a QBIC-based thermal metasurface.

We assume that our metasurface supports a spectral response analogous to Fig. 3(d) for $x$-polarized light. Following the derivation detailed in Appendix A for a more general scenario, it can be shown that the reflection coefficient for such a linearly polarized QBIC satisfying $\gamma_{r}=\gamma_{i}$ is

$$
r(k, \omega)=r_{c}(\omega)+\frac{i}{\left[\omega-\omega_{\text {res }}(k)\right] / \gamma_{r}-i},
$$

where $r_{c}(\omega)$ is a local background scattering coefficient, i.e., due to a Fabry-Perot resonance, slowly varying compared to the resonance features, and $\omega_{\text {res }}(k)$ is the angularly dispersive resonant frequency. Within the frequency range of interest, the local background may be simply approximated by a sinusoidally varying function of $\omega$ :

$$
r_{c}(\omega)=r_{0}-\left(1-r_{0}\right) \cos \left(\frac{2 \pi}{P_{\omega}}\left(\omega-\omega_{1}\right)\right),
$$

where $r_{0}$ is a real-valued, near-unity coefficient, $P_{\omega}$ determines the spectral period of the thin film resonances, and $\omega_{1}$ is a frequency with peak local absorption. The dispersion may be approximated by a Taylor expansion about $k=0$ :

$$
\omega_{\text {res }}(k) \approx \omega_{0}+\frac{b}{2} k^{2},
$$

where the coefficient $b$ is a quantitative measure of the band curvature near the band-edge mode. Finally, the absorption is simply $\mathcal{A}(k, \omega)=1-|r(k, \omega)|^{2}$. By Kirchhoff's law of thermal radiation, the TCMT model for absorption directly provides an analytical form for the thermal emissivity in momentum-frequency space $\varepsilon(k, \omega)=1-R(k, \omega)$, where the reflectance $R(k, \omega)=|r(k, \omega)|^{2}$. To validate this model, Figs. 4(a) and 4(b) show excellent agreement between the spectra calculated by FDTD and this model with appropriately fit values [Fig. 4(a) reproduces Fig. 3(d) for comparison]. Figure 4(c) shows the agreement between FDTD and TCMT spectra for light emitted at $\theta_{x}=0$, and Fig. 4(d) shows the angular profile for light emitted at the band-edge mode. Because of the Wiener-Khinchin theorem [2,4,5], the linewidth of emission, shown in Fig. 4(c), related to the lifetime of the mode $\tau$, directly determines the temporal coherence of the emitted light, namely,

$$
\tau_{c}=\tau=1 / \gamma_{r}
$$

We emphasize, in particular, the fit value $r_{0}=0.9935$ stemming from our calculations, which suggests that, despite the homogeneous material loss in silicon considered in our simulations but neglected in our TCMT model, the broadband, local material loss has negligible effects on this operation. The QBIC-enhanced optical lifetime dominates the spectral features: The QBIC is critically coupled when a very low value of loss is present $\left(\kappa=10^{-2.84}\right)$, which introduces little absorption for nonresonant light. Hence, just as in the case of luminescence [52], the emitted spectrum may be significantly narrowed and the background (unpatterned) emission correspondingly suppressed. This feature improves as the $Q$ factor increases, at the expense of higher sensitivity to design or fabrication errors.

Analogously to the relationship between spectral width and temporal coherence, the angular width of emission, shown in Fig. 4(d), determines the spatial coherence $[68,69]$. For simplicity in highlighting the physics of the nonlocal component of the scattering, we assume that the local scattering is lossless, i.e., $r_{0}=1$, giving the relation

$$
\varepsilon(k, \omega)=\frac{1}{1+\tau^{2}\left(\omega-\omega_{0}-\frac{b}{2} k^{2}\right)^{2}} .
$$

With an analytical form of the optical response in hand, we may derive a closed form expression for the spatial coherence length $L_{c}$. As detailed in Appendix D (see also Refs. [2,67-69]), we arrive at the relation

$$
\mu(x, \omega)=\frac{1}{\operatorname{Im}[\xi(\omega)]} \operatorname{Im}\left[\xi(\omega) \exp \left(-\frac{|x|}{\xi(\omega)}\right)\right],
$$

where

$$
\xi(\omega)=\sqrt{\frac{b \tau / 2}{i-\left(\omega-\omega_{0}\right) \tau}}
$$

is a complex quantity with units of length that characterizes the spatial nonlocality of the response at each frequency. In particular, at the band edge, we have $\xi\left(\omega_{0}\right)=\sqrt{b \tau / 2 i}$, and the form of $\mu(x, \omega)$ is shown in Fig. 4(e) for this scenario. The magnitude of Eq. (19) is half of its maximum at a displacement of approximately $x \approx \sqrt{b \tau}$ (more precisely, its value at $x=\sqrt{b \tau}$ is $[\cos (1)+\sin (1)] / e \approx 0.508)$. The coherence length of such a thermal metasurface at the bandedge frequency is, therefore,

$$
L_{c} \approx \sqrt{b \tau}
$$

It follows that the spatial coherence of the band-edge mode is determined both by the band curvature and by the linewidth of the resonance. Appendix E arrives at the same expression for the coherence length for a parabolic band 

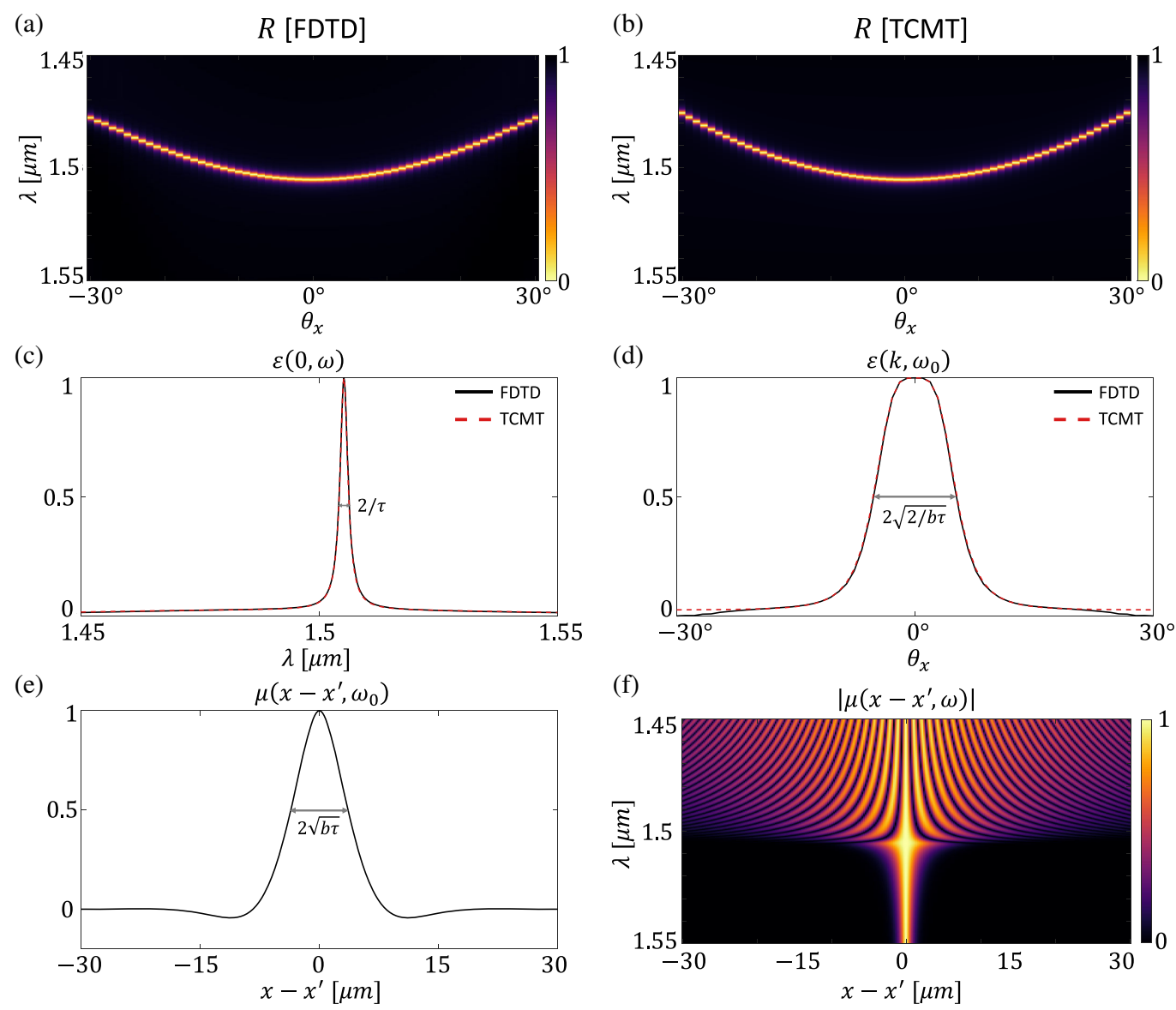

FIG. 4. Temporal and spatial coherence of a QBIC thermal metasurface. (a),(b) Reflectance for $x$-polarized light near a critically coupled QBIC calculated based on FDTD [(a), reproduced from Fig. 3(d)] and TCMT (b). (c) Emissivity at normal incidence, with the linewidth inversely related to the temporal coherence. (d) Emissivity profile at the band-edge frequency, with the angular width given by the spatial coherence. (e) Spectral degree of coherence at the band-edge frequency, with the linewidth characterizing the spatial coherence of the emission. (f) Magnitude of the spectral degree of coherence mapped in the space-frequency domain.

structure by starting from the electric field expression rather than from a CMT description.

Lastly, Fig. 4(f) visualizes $\mu(x, \omega)$ in the space-frequency domain, showing little spatial coherence below the band edge and larger spatial coherence at and above the band edge. Note that this example has a remarkably flat band structure ( $b$ is small), meaning that light at the band edge remains substantially localized due to Bragg reflection, and the spatial coherence is modest. Above the band edge, and for bands with significantly larger values of $b$, the spatial coherence may be much larger. Therefore, the results here suggest that band structure engineering can control to a large extent the degree of lateral localization for a given optical lifetime, while the optical lifetime itself determines the temporal coherence. As explored in Sec. V, the in-plane localization afforded by such a flat band structure enables computationally tractable full-wave simulations of compact aperiodic 3D structures at the expense of spatial coherence; applications requiring higher degrees of spatial coherence should use a QBIC with sharper bands, i.e., a larger value of $b$, than the one investigated in Fig. 4. While we do not pursue advanced dispersion engineering here (beyond finding a sufficiently low value of $b$ ), we note that elaborate control over the band structure using periodic perturbations is demonstrated in Ref. [70], and basic control over the band curvature by tuning the duty cycle and height of a photonic crystal slab is demonstrated in Refs. [54,62] explicitly within the QBIC framework. Beyond QBIC systems, we also note that Ref. [25] recently demonstrated control of the spatial dispersion of thermal emission via polariton-phonon coupling.

\section{CONSTRAINTS ON SPATIAL TAILORING OF THERMAL WAVE FRONTS}

While the dual perturbation introduced in the previous section can tailor the spatial and temporal coherence of thermal emission with simultaneous control of $A, \tau_{c}$, and $L_{c}$, a thermal metasurface must be able to simultaneously control also the local phase and polarization state 
parameters of the emitted light, $\Phi, \chi, \psi$, and $p$, in a spatially varying manner. Such command over thermal emission would enable locally shaped wave fronts with arbitrary polarization. In this section, we outline fundamental constraints on such a spatially varying thermal metasurface stemming from time-reversal symmetry and reciprocity. In particular, we study the constraints on thermal metasurfaces placed over a ground plane, a configuration that enables maximum unidirectional emissivity without complex and sensitive overlap of more than one narrow-band resonance $[71,72]$. Our key finding is that a thermal metasurface placed over a mirror and employing a single QBIC must simultaneously control both local and nonlocal properties of the scattering process, requiring a hybrid approach combining conventional (local) metasurface designs with judicious use of the selection rules governing nonlocal responses.

\section{A. Constraints within temporal coupled mode theory}

To assess the constraints on the scattering of a thermally populated QBIC, we employ TCMT [64-66] to describe the interference between the background (local) scattering process with scattering matrix $C$ and a (nonlocal) QBIC coupled to an external incident state with Jones vector

$$
\left|s_{+}\right\rangle=\left[\begin{array}{l}
s_{x} \\
s_{y}
\end{array}\right]
$$

where $s_{x, y}$ may generally be complex and are normalized such that the inner product $\left\langle s_{+} \mid s_{+}\right\rangle$is the incident intensity. The incident radiation excites a QBIC with resonant frequency $\omega_{0}$ and radiative and nonradiative decay rates $\gamma_{r}$ and $\gamma_{i}$ according to the equation of motion

$$
\frac{d a}{d t}=\left(i \omega_{0}-\gamma_{r}-\gamma_{i}\right) a+\left\langle k^{*} \mid s_{+}\right\rangle,
$$

where $a$ is the complex amplitude of the QBIC resonance, normalized so that $|a|^{2}$ is the stored energy per unit area. The coupling vector $|k\rangle$ indicates how the resonance couples to incident radiation. The reflected wave $\left|s_{-}\right\rangle$is given by

$$
\left|s_{-}\right\rangle=C\left|s_{+}\right\rangle+|d\rangle a,
$$

where $|d\rangle$ is the coupling vector governing how the resonance decays into the output port. The coefficients in Eqs. (23) and (24) are subject to a number of constraints. For example, reciprocity requires that

$$
|k\rangle=|d\rangle,
$$

from conservation of energy we must have

$$
\langle d \mid d\rangle=\frac{2}{\tau},
$$

and combined with Eq. (25) time-reversal invariance dictates that

$$
C\left|d^{*}\right\rangle=-|d\rangle .
$$

Using these constraints, the relation $C=C^{T}$ dictated by reciprocity, and Eqs. (23) and (24), the scattering matrix $\left|s_{-}\right\rangle=S\left|s_{+}\right\rangle$can be written as

$$
S=C+\frac{|d\rangle\left\langle d^{*}\right|}{i\left(\omega-\omega_{0}\right)+\gamma_{r}+\gamma_{i}} .
$$

The scattering matrix as a whole also satisfies the reciprocity condition

$$
S=S^{T}
$$

At the resonant frequency, absorption is unity (and, hence, thermal emission maximized) only when the system satisfies the critical coupling condition $\gamma_{r}=\gamma_{i}$. Detuning from this condition enables control over the emitted amplitude, as seen in Appendix C. Indeed, the radiative and nonradiative $Q$ factors described in Sec. III A are related according to $Q_{r}=\omega_{0} / 2 \gamma_{r}$ and $Q_{l}=\omega_{0} / 2 \gamma_{i}$, and, therefore, these phenomenological parameters within the TCMT are described by parameters that a thermal metasurface may directly control. As shown in the following sections, a thermal metasurface can indeed directly control every phenomenological parameter present in Eq. (28).

The time-reversal constraint in Eq. (27) shows that the scattering of a QBIC to the far field (generally a nonlocal phenomenon) is not independent from the local scattering. Indeed, both the polarization state as well as the phase of the emitted state are constrained by the local scattering $C$. For instance, taking the local scattering to be the one of a perfect mirror

$$
C=\exp \left(i \Phi_{C}\right)\left[\begin{array}{cc}
-1 & 0 \\
0 & -1
\end{array}\right],
$$

where $\Phi_{C}$ is a reference phase, we see upon applying Eq. (27) that the emitted state must satisfy

$$
\exp \left(i \Phi_{C}\right) d_{x, y}^{*}=d_{x, y} ;
$$

i.e., the emitted state is real valued but for the factor $\Phi_{C}$. Or, more generally, the emitted phase $\Phi=\angle d_{x, y}$ is

$$
\Phi=\Phi_{C} / 2
$$

This result has several consequences: First, it implies that, for the emitted state to have an arbitrary polarization state, the local scattering must be birefringent to break the condition $\angle d_{x}=\angle d_{y}$. We generally desire minimal local 
absorption, since we aim for thermal emission across narrow bandwidths around the Fano resonance, meaning that the local scattering may not have any dichroism; the local birefringence is the only parameter that may lift the polarization state from the equator of the Poincaré sphere, where $\angle d_{x}=\angle d_{y}$. For instance, to achieve pure circularly polarized emitted light, the direct scattering matrix must behave as a half wave plate:

$$
C=\exp \left(i \Phi_{C}\right)\left[\begin{array}{cc}
-1 & 0 \\
0 & 1
\end{array}\right]
$$

and so, by Eq. (27), the emitted state must follow

$$
\begin{aligned}
& \exp \left(i \Phi_{C}\right) d_{x}^{*}=d_{x}, \\
& \exp \left(i \Phi_{C}\right) d_{y}^{*}=-d_{y},
\end{aligned}
$$

allowing $\angle d_{x}=\Phi_{C} / 2$ and $\angle d_{y}=\left(\Phi_{C} \pm \pi\right) / 2$, corresponding to a circularly polarized state at a pole of the Poincaré sphere, where $\angle d_{x}-\angle d_{y}=\mp \pi / 2$.

The second consequence of Eq. (32) is that the phase of the emitted state is directly controlled by the local scattering phase; i.e., the emitted phase is half the phase that would be imparted by the local component of the metasurface if the QBIC were absent. Overall, this time-reversal constraint shows that a thermal metasurface based on a QBIC must vary the local scattering properties; for complete control of the thermally emitted light, it is not sufficient to vary the perturbation according to the selection rules as done in recent nonlocal metasurfaces showing spatial control of Fano resonances [53,54] and even wavefront-selective Fano resonance [57].

\section{B. Visualization of the constraints with schematic scattering matrices}

Finally, we illustrate how these concepts, as well as the reciprocity constraint in Eq. (29), apply to the goal of unidirectional emission with a single specified spin. Figure 5 sketches the energy exchange between two blackbodies at the same temperature, mediated by four distinct metasurface mirrors that support QBICs at the operating wavelength and angles of incidence enabling exchange of energy between $A$ and $B$. Figure 5(a) provides the definitions and conventions used for the schematic depictions of idealized scattering matrices [the example of a perfect mirror is given in Fig. 5(b)], and Fig. 5(c) summarizes the behavior of the four metasurfaces studied in Figs. 5(d)-5(k). The scattering matrices apply to electric fields in a circular polarization basis across a continuous range of momenta:

$$
\mathbf{E}(k)=\left[\begin{array}{c}
\mathbf{E}_{L}(k) \\
\mathbf{E}_{R}(k)
\end{array}\right] .
$$

The first metasurface [Figs. 5(d) and 5(e)] is a device that supports an absorptive QBIC born of interference with a local response that has no phase gradient or birefringence, equivalent to a specular mirror. In the absence of a QBIC, its scattering matrix is given by Eq. (30) and is shown in Fig. 5(b). This schematic represents the sparse matrix of scattering magnitudes due to the device, where the gray background refers to scattering events forbidden by conservation of linear momentum, and, hence, of magnitude 0 , and orange lines refer to scattering events satisfying conservation of linear momentum ( $k_{\text {out }}=k_{\text {in }}+m k_{G}$, where $k_{\text {out }}$ is the horizontal output momentum, $k_{\text {in }}$ is the horizontal input momentum, $k_{G}=2 \pi / P$ is the quasimomentum associated with a period $P$, and $m$ is the diffraction order). The solid orange lines refer to scattering efficiencies of unity (here, $k_{\text {out }}=k_{\text {in }}$ such that the reflected spin is inverted), while the dashed orange lines refer to scattering efficiencies of zero $\left(k_{\text {out }}=k_{\text {in }}\right.$ such that the reflected spin is preserved). Reciprocity [Eq. (29)] requires that these schematics have a mirror symmetry across the black dashed line.

The first metasurface differs from a specular mirror by the addition of a QBIC with a nonlocal phase gradient. This addition is represented by a purple dot, whose magnitude may represent any number between 0 (perfect absorption) and 1 (perfect reflection). Using the approach demonstrated in Ref. [56], a nonlocal phase gradient may be used to shift the band-edge mode in $k$ space and, therefore, the angle at which the thermal metasurface emits. Per Eqs. (27) and (28), such a nonlocal phase gradient is not independent of the local response. In particular, since the absorption due to a QBIC is born of interference with the local (background) matrix, in the schematic in Fig. 5(e), it may be placed only along the orange dashed line. In the absence of the nonlocal phase gradient, it would be located at $\left(k_{\text {out }}, k_{\text {in }}\right)=(0,0)$, but here we consider the QBIC having been shifted to $\left(k_{\text {out }}, k_{\text {in }}\right)=\left(k_{\mathrm{nl}}, k_{\mathrm{nl}}\right)$, where $k_{\mathrm{nl}}$ is the momentum added by the nonlocal phase gradient.

Despite this shift in $k$ space, it is readily apparent that reciprocity forbids unidirectional emission, consistent with the discussion in Sec. III A. For instance, for a device aiming to absorb RCP light with $k_{\mathrm{nl}}<0$ [marked by an encircled 1 in Fig. 5(e)], a reciprocal event (marked by an encircled $1^{*}$ ) exists corresponding to absorption of LCP with $k_{\mathrm{nl}}>0$. In the schematic, the absorption is understood by finding the angle at which the QBIC exists along the $\theta_{\text {in }}$ direction, and the polarization state being absorbed is understood by the second letter labeling the quadrant (the one labeled with the $*$, denoting a state traveling in the downward direction). For instance, located in the $L R^{*}$ quadrant in Fig. 5(e), the encircled 1 corresponds to 


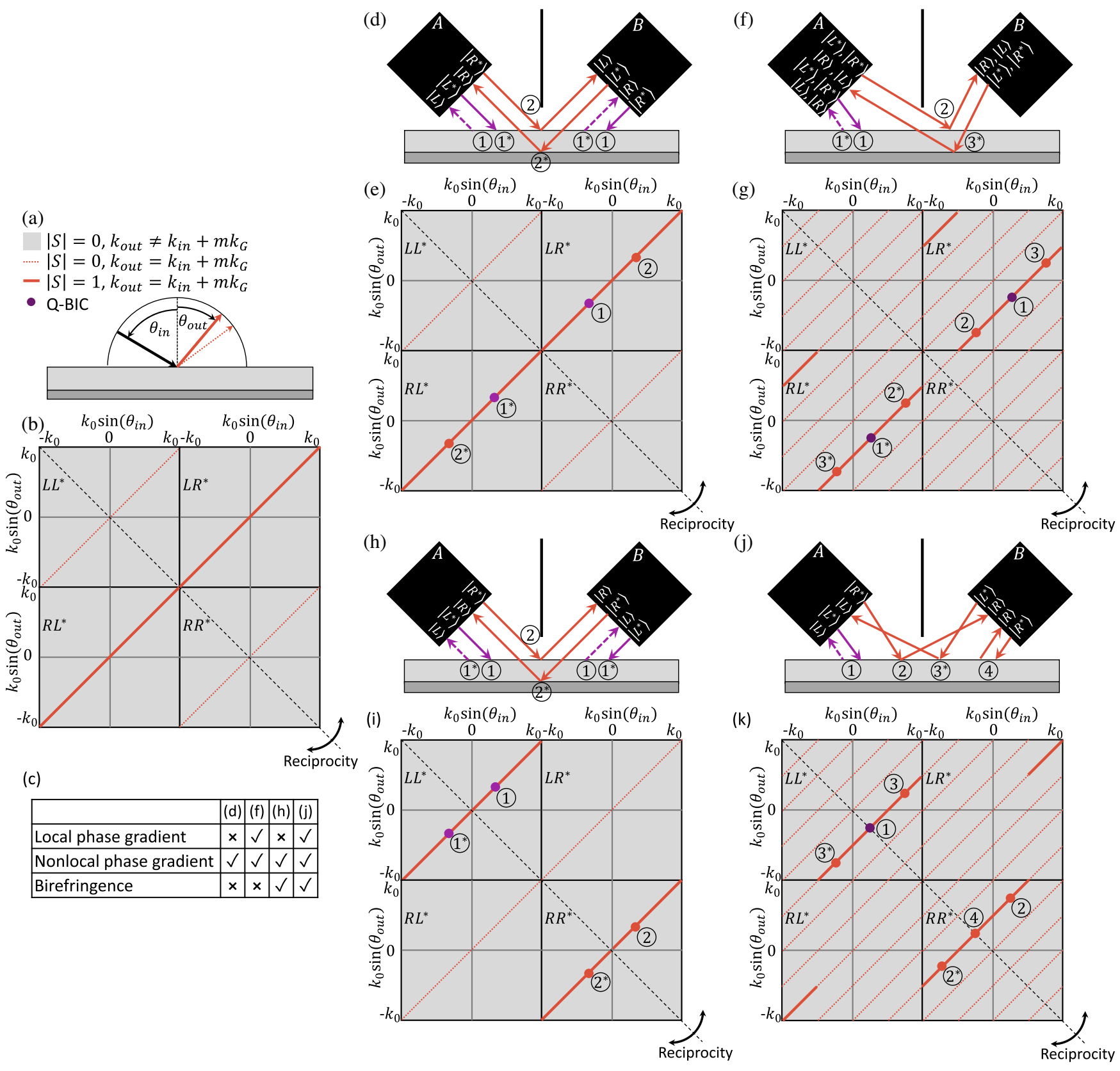

FIG. 5. Reciprocity and spin-selective unidirectionality. (a) Definitions and conventions for the continuous scattering matrices as in (b), which depicts the behavior of a specular mirror (which conserves linear momentum and flips the spin upon reflection). (c) Summary of the behavior of the four devices studied in (d)-(k). (d) Schematic showing two blackbodies, $A$ and $B$, exchanging energy through a locally specular mirror with a nonlocal QBIC, depicted in (e) as a purple dot placeable anywhere along the specular response (solid orange line) via a nonlocal phase gradient. Reciprocity (symmetry about the black dashed line) forbids the existence of unidirectional emission. Similarly, (f) and (g) show the case of a local phase gradient but no nonlocal phase gradient, enabling unidirectional emission but not spin selectivity. To achieve spin selectivity, birefringence must be added, shown in (h) and (i) for the case of a birefringence local response with only a nonlocal phase gradient. However, reciprocity forbids unidirectionality except at normal incidence in this case. Finally, $(\mathrm{j})$ and $(\mathrm{k})$ show the case with a local phase gradient, nonlocal phase gradient, and birefringence, enabling spin-selective unidirectional thermal emission.

absorption of $\left|R^{*}\right\rangle$ at an angle $\theta_{\text {in }}<0$, while the reciprocal event corresponds to absorption of $\left|L^{*}\right\rangle$ at an angle $\theta_{\text {in }}>0$. Upon time reversal, these absorption events correspond to emission events satisfying time-reversal symmetry [60]:

$$
\mathbf{E}(k)=\mathbf{E}^{*}(-k) .
$$

In the schematic, the emission may be understood by looking at the location along the $\theta_{\text {out }}$ axis and the first letter 
marking the quadrant (without the label *, corresponding to upward-traveling states). For instance, the encircled 1 under time reversal denotes the emission of $|L\rangle$ to an angle $\theta_{\text {out }}<0$, while the encircled $1^{*}$ under time reversal denotes the emission of $|R\rangle$ to an angle $\theta_{\text {out }}>0$. That is, as depicted in Fig. 5(d), the emission and absorption events (purple) are completely described by the process 1 and its reciprocal copy, $1^{*}$. Because of the existence of this distinct reciprocal copy, this device cannot unidirectionally emit light even if it treats LCP and RCP differently. The emission of this device resembles the results of Ref. [38], for example.

The second metasurface [Figs. 5(f) and 5(g)] has a local phase gradient but no birefringence, and a QBIC is applied with a nonlocal phase gradient. In this case, many diffraction orders $m$ are supported, but the idealized case in Fig. 5(g) supports only a single diffraction order with unity reflectance. Without birefringence, the usual spin inversion occurs upon reflection, but, due to the local phase gradient, the outgoing angle satisfies $k_{\text {out }}=k_{\text {in }}-k_{G}$. As before, a QBIC may be placed along this diffraction order. In this case, it exists at the position $\left(k_{\text {out }}, k_{\text {in }}\right)=\left(k_{G} / 2, k_{G} / 2\right)$, corresponding to local retroreflection. It is apparent that the process 1 and its reciprocal copy $1^{*}$ represent emission of light to the same outgoing angle $\theta_{\text {out }}$ but to polarization states of $|L\rangle$ and $|R\rangle$, respectively. The introduction of a local phase gradient does interestingly enable unidirectionality, but without birefringence this cannot be achieved in a spin-selective manner (the result here is unidirectional thermal emission with a linear polarization). Note that using a different nonlocal phase gradient would move the QBIC off the retroflection condition and destroy the unidirectionality.

The third metasurface [Fig. 5(h)] adds birefringence to the case in Fig. 5(d), providing a nonlocal phase gradient but no local phase gradient, as described by the local scattering matrix in Eq. (33). In this case, the local response preserves the spin upon reflection, moving the solid orange line to the appropriate quadrants compared to a specular mirror. Here, using a chiral QBIC, it is possible to achieve spin selectivity. That is, the process 1 and its reciprocal copy $1^{*}$ both represent emission of light to the state $|L\rangle$, which are, therefore, independent of the $\mathrm{RCP}$ response due to the birefringence. However, as in the previous cases, the presence of the reciprocal copy means that unidirectionality is forbidden: Light is emitted to a state $|L\rangle$ to two equal and opposite angles. Notably, however, if the nonlocal phase gradient is removed (or set to 0 ), the processes 1 and $1^{*}$ merge. In other words, process 1 is then its own reciprocal copy, because it exists at the intersection of the orange solid line and the black dashed line. Consequently, spin-selective unidirectional emission may be achieved in such a system but only to normal incidence.

Finally, to generalize this achievement to any emission angle, our fourth metasurface [Figs. 5(j) and 5(k)] includes a local phase gradient metasurface along with birefringence (e.g., a Pancharatnam-Berry or geometric phase metasurface $[9,12])$ and then places the QBIC at the retroreflection condition using a nonlocal phase gradient. This configuration inherits the unidirectionality to an arbitrary angle stemming from the local phase gradient, as seen in the second device [Figs. 5(f) and 5(g)], as well as the spin selectivity afforded by the birefringence, as seen in the third device [Figs. 5(h) and 5(i)]. As depicted in Fig. 6(j), this behavior is consistent with the second law of thermodynamics concerning exchange of energy between bodies $A$ and $B$ : since RCP light coming from $B$ is retroreflected back to $B$ (marked by an encircled 4 ) and LCP light coming from $A$ is completely absorbed (marked by an encircled 1 ), these two channels do not exchange energy between $A$ and $B$ and, therefore, are independent. Meanwhile, the remaining processes (encircled 2 and 3) represent reciprocal channels of energy exchange between $A$ and $B$.

To summarize the fundamental results of this section, time-reversal symmetry generally requires that a thermal metasurface can achieve spin-selective unidirectional emission to an arbitrary angle of choice only through a suitable combination of a birefringent local phase gradient and a nonlocal phase gradient. The birefringence makes the LCP and RCP channels independent in the far field, enabling a chiral structure to absorb a single spin without violating reciprocity. This structure enables spin-selective unidirectional emission at normal incidence in the absence of local and nonlocal phase gradients, because the absorption process is its own reciprocal (retroreflection). Extending this property to any off-normal emission angle requires a local phase gradient to shift the retroreflection angle to the desired angle and a nonlocal phase gradient to place the QBIC precisely at the retroreflection condition.

Last, to further clarify this general result, we compare it to the behavior of the metasurface in Fig. 3. In Sec. III A, we note that the band diagram of the device is fundamentally constrained by reciprocity to be symmetric about normal incidence, with eigenfrequencies coming in pairs $\omega_{i}(k)=\omega_{i}(-k)$. Notably, this pairing of eigenfrequencies is true in any reciprocal system, including both Fig. 3 and all the devices in Fig. 5. However, quite interestingly, this property translates onto a symmetric emission pattern in Fig. 3(d) but not in Fig. 5(j). While the underlying band structure of any reciprocal system satisfies $\omega_{i}(k)=\omega_{i}(-k)$, the far-field coupling of these modes must simply satisfy Eq. (29), which we may write in the basis of Eq. (35) as

$$
S_{i j}\left(k_{1}, k_{2}\right)=S_{j i}\left(-k_{2},-k_{1}\right)
$$

Here, the indices refer to the polarization state, the first argument is the incoming wave number, and the second argument is the outgoing wave number. As argued schematically above on the basis of Fig. 5, it is apparent from Eq. (37) that any modal absorption (say, at an angle 
$k_{\text {in }}=k_{1}$ and polarization $i$ ) necessarily comes with a reciprocal partner at $\left(k_{\text {in }}=-k_{2}\right.$ and polarization $\left.j\right)$. Unidirectional absorption is, therefore, possible only when a single absorption event occurs such that $k_{1}=-k_{2}$ and $i=j$, which is the retroreflection condition in the appropriate polarization basis (Fig. 5 specifically refers to circular polarization). Note that, when there are several modes contributing to absorption, the total absorption need not be the same at $k_{1}$ and $k_{2}$-here, we focus on a single mode.

Since QBICs are born of interference with the background scattering of the surface, in periodic structures, they may emerge only at $k_{2}=k_{1}+m k_{G}$, where $k_{G}$ is the phase gradient of the device and $m$ is the diffraction order. Considering that for unidirectional absorption $k_{1}=-k_{2}$, conservation of momentum thereby requires that $2 k_{2}=m k_{G}$. When $m=0$ (e.g., specular reflection), therefore, unidirectionality may occur only at normal incidence, where $k_{1}=-k_{2}=0$, consistent with the device in Fig. 3 . Yet, for any other diffraction order, unidirectional thermal emission can occur at the arbitrary angle provided that the supported QBIC emerges at the retroflection condition $k_{2}=-k_{1}=m k_{G} / 2$. Hence, quite interestingly, we can generally state that a critically coupled QBIC unidirectionally emits with outgoing momentum half of the one contributed by the local phase gradient.

\section{THERMAL METASURFACE DESIGNS}

Guided by the general findings of the previous section, we can now construct a library of unit cells for the demonstration of various functionalities enabled by thermal metasurfaces. In the following, after developing this general library, we use it to show that an arbitrary elliptical state may be emitted to normal incidence by suitably engineering the geometrical parameters governing the local and nonlocal responses. Then, we numerically demonstrate unidirectional emission with desired spin by locally varying the geometric phase while adjusting the nonlocal scattering accordingly. By spatially varying the emission angle, we then demonstrate thermal metasurfaces producing quasimonochromatic light focused to a desired point in the far field. Finally, by varying orientation of the off-normal emission azimuthally, we demonstrate thermal metasurfaces with control of both spin and orbital angular momentum of emitted light.

\section{A. Metaunit library for thermal metasurfaces}

We begin with a discussion of the ideal features of a metaunit library for thermal metasurfaces: First, the device must have a local element controlling both the local scattering phase and the relative phase between $x$ and $y$ polarizations, i.e., the degree of birefringence. Second, the device should support a symmetry-protected BIC as unaffected as possible by the geometric perturbations that govern the local scattering. At minimum, the $Q$ factor should remain infinite regardless of the chosen phase and degree of birefringence; there should be nonzero scattering only in the presence of additional perturbations. This constraint generally excludes QBICs supported by monatomic lattices and suggests instead the use of QBICs folded into the continuum by a period-altering perturbation [62]. Ideally, the resonant frequency should be invariant to, for instance, the in-plane orientation of the structural birefringence, but at minimum the resonant frequency should be adjustable by both the local response and perturbation such that a constant resonant frequency may be achieved for any local response. Third, in order to access circular polarizations, the device must be optically chiral. This constraint suggests (though, due to the mirror plane, does not require) placing the symmetry-breaking perturbation at a distinct plane from the local response. Fourth, the symmetryprotected BIC should be controlled by a perturbation with control over both the eigenpolarization and $Q$ factor; this process may be guided by selection rules to choose an appropriate perturbation for the chosen mode [54].

Based on these considerations, we now demonstrate an implementation of a metaunit library for thermal metasurfaces. These guidelines suggest starting with a monatomic lattice of elliptical dielectric pillars with complete control over the phase and polarization of locally scattered light, as, e.g., demonstrated in Ref. [9], and then adding a dimerizing perturbation at a different plane. Such an unperturbed lattice satisfies the first requirement above; the addition of the dimerizing perturbation satisfies the second requirement by accessing a QBIC folded into the continuum by the period doubling; and placing the perturbation at a separate plane satisfies the third requirement regarding chirality. The fourth requirement may be satisfied by using a perturbation with a $p 2$ space group, allowing simultaneous control over the scattering rate and polarization angle scattered at the interface where the perturbation is placed $[53,54]$.

As seen in Fig. 6(a), the example device is a monatomic array of doped silicon elliptical pillars sitting on a perturbed doped silicon slab within a $p 2$ space group. This structure sits atop a perfect electric conducting (PEC) ground plane, and a low-index lossless dielectric fills the volume between the PEC and the silicon structure [Fig. 6(b)]. The monatomic array controls the local scattering (phase and polarization), and so we refer to it as the local element or the monomer, with height $H$, length $L$, and width $W$ having subscripts $m$. Since, in general, the in-plane orientation angle of the dielectric pillars $\theta$ is varied, we target a symmetry-protected BIC whose field profile overlaps so as to be minimally affected by such rotation. A mode with this property is the transverse-magnetic mode (characterized primarily by an electric field pointing out of plane) that has its electric field concentrated within the high-index pillars [Fig. 6(c)]. We next refer to the selection rules [54] to determine the appropriate $p 2$ perturbation that accesses this mode. The perturbation is a set of thin 

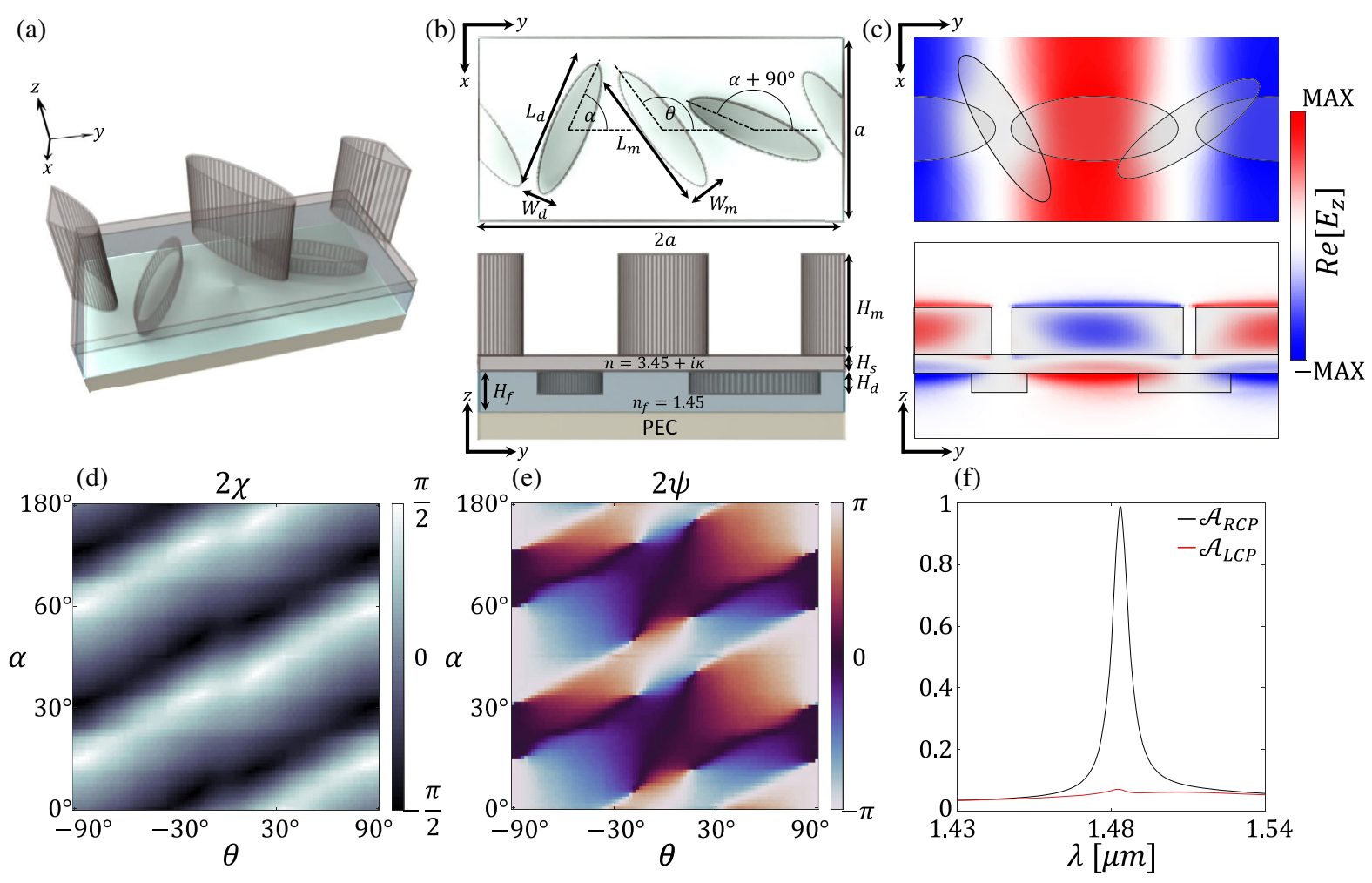

FIG. 6. Metaunit library of a thermal metasurface. (a) Perspective view of a structured silicon film sitting atop a PEC with a dielectric filler in between. The top silicon elliptical pillars are monomers, and the bottom are dimers. (b) Top and side views of the unit cell in (a), defining the geometric and material parameters. (c) Cross sections of an example quasi-BIC supported by this structure and controlled by the dimer. (d),(e) The latitude and longitude on the Poincare sphere of the quasi-BIC in (c) of the absorbed polarization state as the geometric parameters $\theta$ and $\alpha$ are varied. (f) Circularly dichroic absorption spectra for the geometry depicted in (c).

elliptical pillars centered between the local pillars and oriented at angles $\alpha$ and $\alpha+90^{\circ}$ (forming a dimer). These features control the symmetry-breaking response governing the nonlocal QBIC, and so we refer to it as the nonlocal element or the dimer (with height $H$, length $L$, and width $W$ having subscripts $d$ ).

As shown in Sec. III A, the QBIC may be critically coupled by adjusting both the magnitude of the perturbation $\left(H_{d}, L_{d}, W_{d}\right)$ to match the local amount of loss introduced by doping $(\kappa)$. As usual for nondegenerate Fano resonances, this absorption is dichroic: The device perfectly absorbs one polarization state but not the orthogonal state. By choosing the monomer's birefringence to be the one of a half wave plate, i.e., described by Eq. (33), we show that the absorbed polarization state may be any arbitrary elliptical state. Figures 6(d) and 6(e) show, as a function of the orientation angles of the monomer $\theta$ and dimer $\alpha$, the latitude $2 \chi$ and longitude $2 \psi$ on the Poincaré sphere describing the absorbed, and, hence, thermally emitted, state. It is clear that by simultaneously varying $\theta$ and $\alpha$ any emitted polarization state is possible. This behavior may be roughly understood as follows: The dimer scatters the QBIC near the PEC interface to a linear polarization oriented at an angle $\phi \approx 2 \alpha$, which then travels through the local element adjusted by the Jones matrix

$$
\begin{aligned}
J= & \exp \left(i \Phi_{C} / 2\right)\left[\begin{array}{cc}
\cos (\theta) & -\sin (\theta) \\
\sin (\theta) & \cos (\theta)
\end{array}\right]\left[\begin{array}{ll}
i & 0 \\
0 & 1
\end{array}\right] \\
& \times\left[\begin{array}{cc}
\cos (\theta) & \sin (\theta) \\
-\sin (\theta) & \cos (\theta)
\end{array}\right]
\end{aligned}
$$

representing the single-pass behavior of the local element as a quarter wave plate layer oriented at an angle $\theta$. The emitted state has the form

$$
\begin{aligned}
|d\rangle & =J\left[\begin{array}{c}
\cos (2 \alpha) \\
\sin (2 \alpha)
\end{array}\right] \\
& =\exp \left(i \Phi_{c} / 2\right) \frac{1+i}{2}\left[\begin{array}{c}
\cos (\phi)+i \cos (2 \theta-\phi) \\
\sin (\phi)+i \sin (2 \theta-\phi)
\end{array}\right] .
\end{aligned}
$$

More rigorously, we can describe this system with TCMT. The background scattering matrix in this case is

$$
C=\exp \left(i \Phi_{C}\right)\left[\begin{array}{cc}
\cos (2 \theta) & \sin (2 \theta) \\
\sin (2 \theta) & -\cos (2 \theta)
\end{array}\right] .
$$

We then look for solutions for $|d\rangle$ satisfying Eqs. (26) and (27). Guided by the form expected by Eq. (39), we find the solution 


$$
|d\rangle={\sqrt{2 \gamma_{r}}}^{1+i} \frac{i}{2}\left[\begin{array}{c}
\cos (\phi)+i \cos (2 \theta-\phi) \\
\sin (\phi)+i \sin (2 \theta-\phi)
\end{array}\right] e^{i \Phi_{c} / 2},
$$

where $\phi \approx 2 \alpha$ is the linear polarization scattered by the dimer. Since for our initial demonstrations of thermal metasurfaces we focus on geometric phase engineering

$$
S=\frac{i}{2}\left[\begin{array}{c}
{[\sin (\Delta)-i \sin (\phi)]^{2}} \\
\frac{1}{2}[\sin (2 \Delta)+\sin (2 \phi)-2 i \sin (2 \theta)]
\end{array}\right.
$$

where $\Delta=\phi-2 \theta$. Appendix A provides the full general form of the scattering matrix. This matrix is symmetric and singular, meaning it must have a zero eigenvalue. The eigenvector associated with the zero eigenvalue is

$$
|e\rangle=\left[\begin{array}{c}
\cos (\phi)-i \cos (\phi-2 \theta) \\
\sin (\phi)+i \sin (\phi-2 \theta)
\end{array}\right]
$$

implying that the emitted state is [60]

$$
\left|e^{*}\right\rangle=\left[\begin{array}{c}
\cos (\phi)+i \cos (2 \theta-\phi) \\
\sin (\phi)+i \sin (2 \theta-\phi)
\end{array}\right]
$$

This state may be written in the more suggestive form

$$
\left|e^{*}\right\rangle=\left[\begin{array}{cc}
\cos (\psi) & -\sin (\psi) \\
\sin (\psi) & \cos (\psi)
\end{array}\right]\left[\begin{array}{c}
\cos (\chi) \\
i \sin (\chi)
\end{array}\right],
$$

which is a parameterization of an elliptical polarization with $\psi=\theta$ and $\chi=\theta-\phi \approx \theta-2 \alpha$. The TCMT model agrees well with the full-wave simulations in Figs. 6(d) and $6(\mathrm{e})$.

Notably, $\chi$ is roughly constant for contours following $2 \alpha \approx \theta+\beta$, where $\beta$ is some constant angle. In particular, for $\beta=45^{\circ}$, the device emits RCP light [Fig. 6(f)]. But for $\beta$ slightly off from this condition, it absorbs a state nearly fully circularly polarized $(\chi \approx \pi / 4)$ but with $\psi=\theta$, i.e.,

$$
\left|e^{*}\right\rangle \approx \frac{1}{\sqrt{2}}\left[\begin{array}{cc}
\cos (\theta) & -\sin (\theta) \\
\sin (\theta) & \cos (\theta)
\end{array}\right]\left[\begin{array}{l}
1 \\
i
\end{array}\right]=\frac{1}{\sqrt{2}} e^{-i \theta}\left[\begin{array}{l}
1 \\
i
\end{array}\right],
$$

meaning that there is a geometric phase associated with coupling to this QBIC, $\Phi_{\text {geo }}=-\theta$, and, therefore, a device composed of the structures in Fig. 6 may emit narrow-band circularly polarized light at any locally defined phase. For any other polarization, such as linear, we again note that $\Phi_{C}$ in Eq. (38) may be adjusted by judicious control of $L_{m}$ and $W_{m}$ (see Appendix A). Restricting ourselves to the convenient sublibrary targeting circularly polarized light controlled by a geometric phase allows us to keep $L_{m}$ and $W_{m}$ fixed. for circularly polarized emitted light, the value of $\Phi_{c}$ is an arbitrary constant; we set it to 0 for now. Appendix A shows that, by including it (by varying the propagation phase), we can generalize the present results to any arbitrary polarization state. The resulting scattering matrix when $\omega=\omega_{0}$ and $\gamma_{i}=\gamma_{r}$ is then

$$
\begin{gathered}
\frac{1}{2}[\sin (2 \Delta)+\sin (2 \phi)-2 i \sin (2 \theta)] \\
{[\cos (\Delta)+i \cos (\phi)]^{2}}
\end{gathered},
$$

\section{B. Example thermal metasurfaces}

As a first demonstration, Fig. 7 shows two devices with unidirectional spin-selective emission obtained by implementing a phase gradient based on geometric phase engineering. In the first device [Fig. 7(a)], the phase gradient is applied in the $x$ direction, which is orthogonal to the dimerization direction. In the second example [Fig. 7(b)], the phase gradient is applied in the $y$ direction, i.e., in the same direction as the dimerization. The geometrical parameters for these devices are given in Appendix F. Connecting to the general discussion around Fig. 7, we notice that the nonlocal parameter $\alpha$ varies at half the pace of the local parameter $\theta$; i.e., the dimer pattern (dark gray) has twice the period of the monomer pattern (light gray) in Figs. 7(a) and 7(b), implying that the resulting metasurfaces support twice as many diffraction orders as expected from the purely local response. This result implies that for the geometries in Figs. 7(a) and 7(b) the diagrams in Fig. 5 sketch only the diffraction orders associated with the local response or, equivalently, depict only the even diffractive orders.

The spectral emissivity along the $\theta_{x}$ direction for the first device is shown in Fig. 7(c) for RCP and Fig. 7(d) for LCP. These plots are produced by recording the absorption of circularly polarized plane waves incident to the device at each angle $\theta_{x}$ (calculated using the finite difference time domain method using a commercial software by Lumerical Solutions) and then invoking Kirchhoff's law. A band-edge mode with near-unity emissivity arises near $\lambda=1.4 \mu \mathrm{m}$ and $\theta_{x}=18^{\circ}$ for RCP, but it is absent for the symmetric band at $\theta_{x}=-18^{\circ}$ for LCP. The angular emission profile at the band-edge frequency is shown in Fig. 7(e), showing a stark contrast for LCP and RCP and confirming the largely spin-selective and directional features of the thermal emission. The unwanted sidelobes are due to other modes supported by the structure, but they (i) are not near unity in emissivity and (ii) do not show a marked discrepancy between LCP and RCP. Indeed, they show the conventional behavior of geometric phase metasurfaces: For every LCP peak, there is an RCP peak at an equal and opposite angle. Further work is needed to reduce the prevalence of these side peaks if the desired application calls for it. In general, 

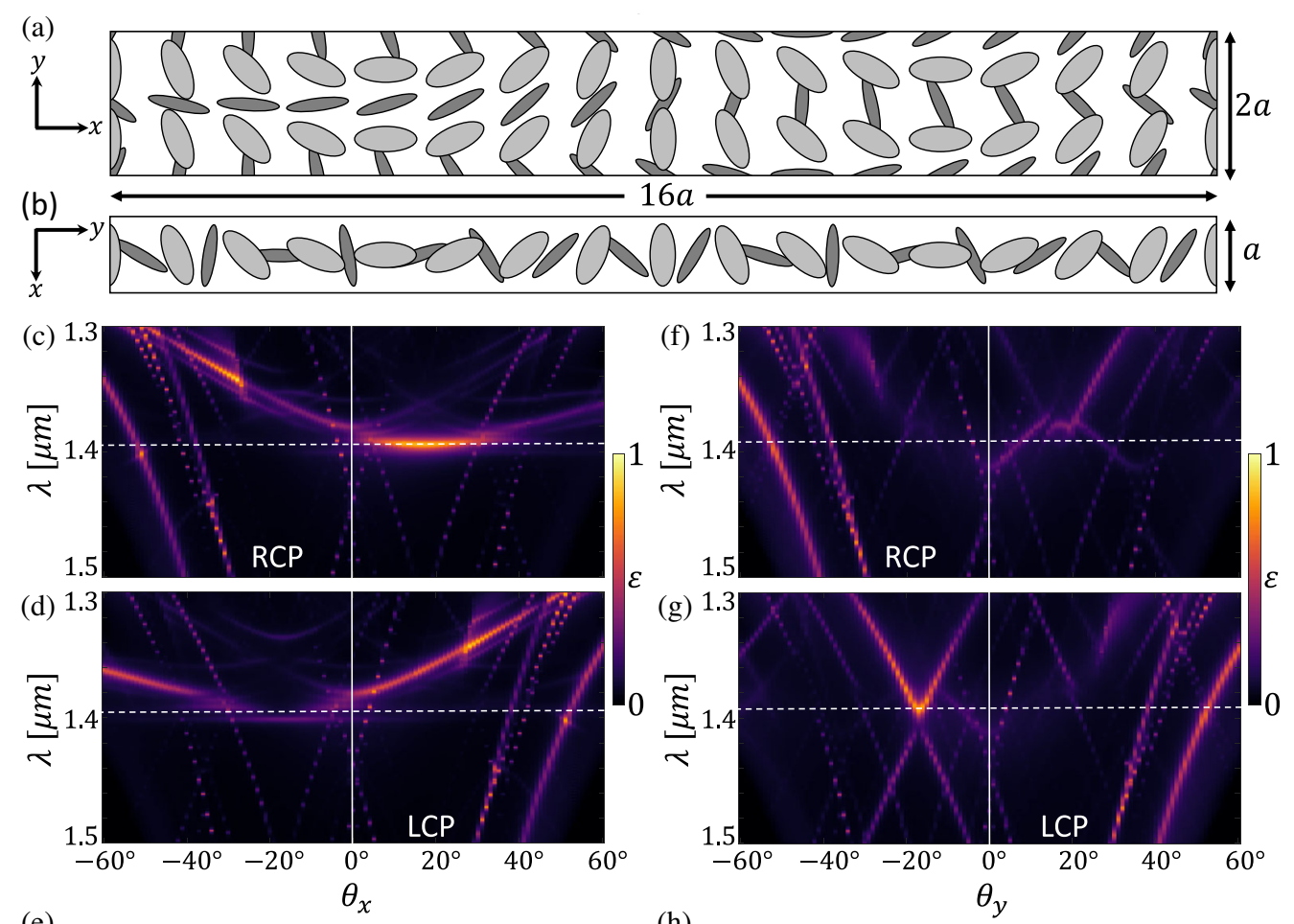

(e)
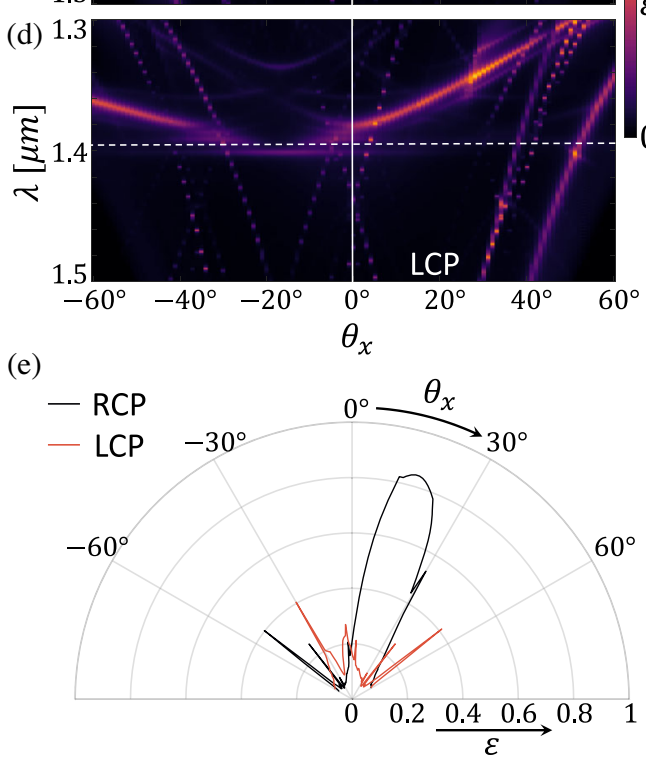

(h)

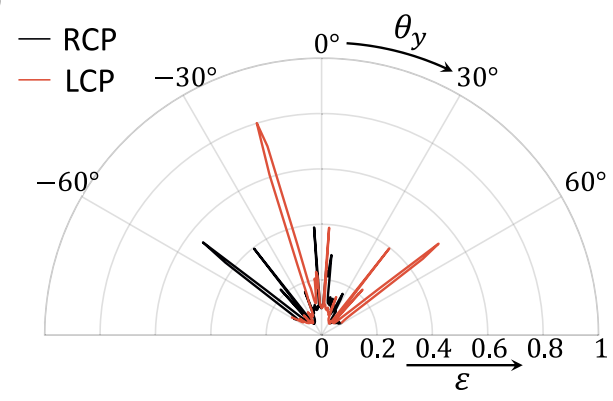

FIG. 7. Selective and directional emission from a thermal metasurface. (a) The superperiod of a thermal metasurface designed to emit RCP light at non-normal incidence, with low spatial coherence. (b) A superperiod designed to emit LCP light at non-normal incidence, with high spatial coherence. (c),(d) RCP and LCP emission maps for the device in (a) as a function of the wavelength and altitude in the $x$ direction, $\theta_{x}$. (e) Emission patterns for the device in (a) at the wavelength of the quasi-BIC's band edge [dashed contours in (c) and (d)]. The band is flat near the band edge, meaning the spatial coherence is low and the emitted lobe is broad. (f), ( $\mathrm{g}$ ) RCP and LCP emission maps for the device in (b) as a function of wavelength and altitude in the $y$ direction, $\theta_{y}$. (h) Emission patterns for the device in (b) at the wavelength of the quasi-BIC's band edge [dashed contours in (f) and (g)]. The band is not flat near the band edge, meaning the spatial coherence is high and the emitted lobe is narrow.

we find that, since they are not specifically engineered by the thermal metasurface geometrical parameters, they tend to be washed out when considering thermal metasurfaces with aperiodic wave fronts, as considered in the following examples.

Next, we show that focused thermal emission with selected spin is possible by varying the emission angle across the device. Shown in Fig. 8(a), the orientation of the monomer and dimer in the array is varied to match the hyperbolic phase function required by a cylindrical metasurface lens focusing along the $x$ direction. The thermal behavior of the device is then studied with full-wave simulations by recording the absorption of energy emanating from circularly polarized point sources placed at arbitrary locations in the $x, z$ plane. By the fourth modal radiation law [60], the resulting map of absorption is equivalent to the intensity pattern of emitted light near the designed focal spot of the device. Figures 8(b) and 8(c) map the spectral emissivity along the optical axis and across the focal plane, respectively, showing that light emitted at the band-edge frequency is indeed focused. Figures 8(d) and 8(e) depict the cross sections marked in Figs. 8(b) and 8(c) for the band-edge frequency and a nonresonant frequency, respectively, showing focused thermally emitted RCP light at the band-edge frequency and negligible emission for the other frequency. We note that, despite the presence of the side peaks in Fig. 7, their impact is washed out here due to (i) their comparably sharper 
(a)

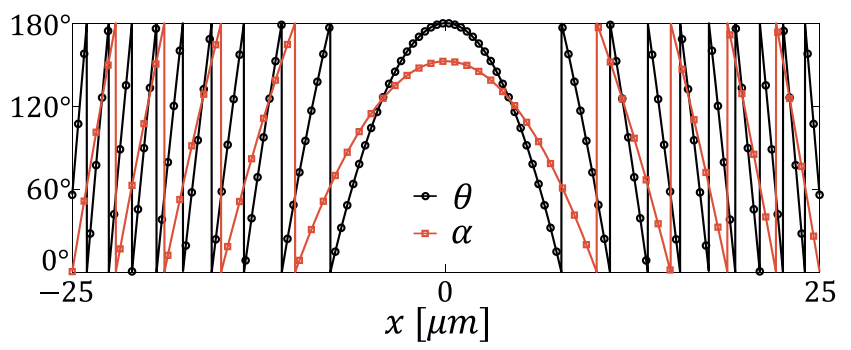

(b)

(d)

(e)
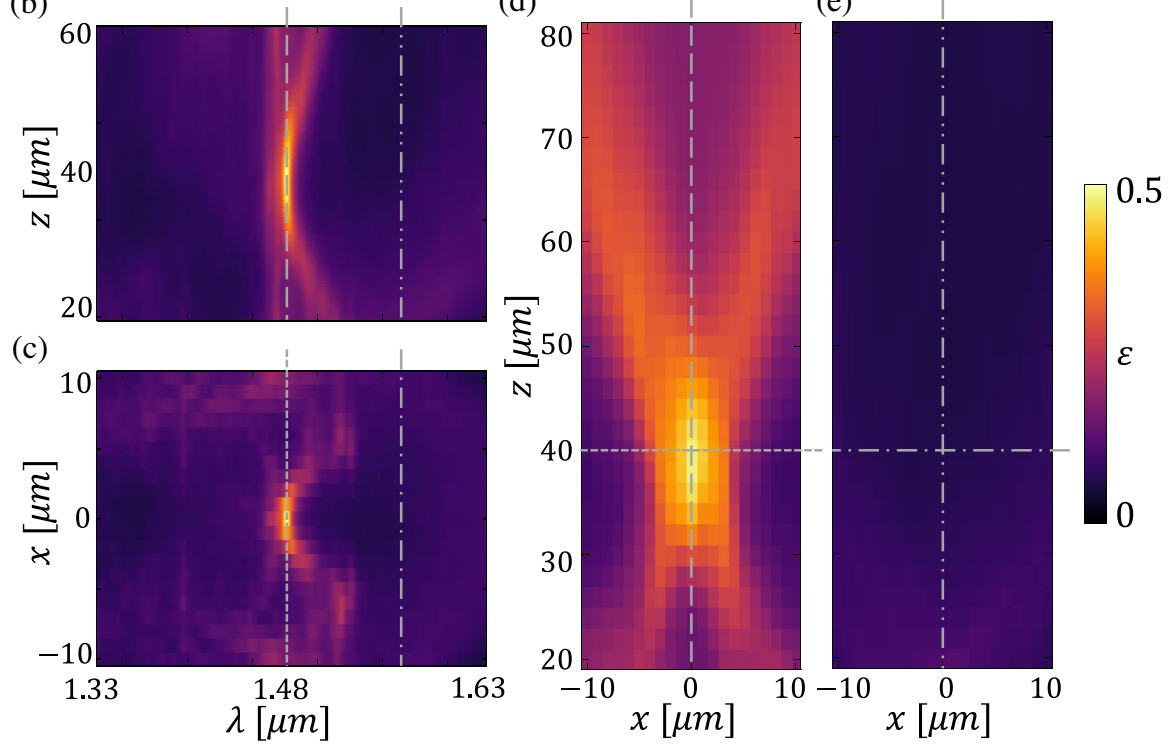

FIG. 8. Thermal metasurface focusing narrow-band RCP light emission. (a) Angular parameters of the thermal metasurface producing focused thermal emission. (b) RCP emission map as a function of the wavelength and position $z$ along the optical axis ( $x=0$ ). (c) RCP emission map as a function of the wavelength and position $x$ at the focal plane $(z=40 \mu \mathrm{m})$. (d),(e) Profile of emitted light near the focal spot of the device for the band-edge wavelength (d) and nonresonant wavelength (e).

angular dispersion and (ii) only contributing at a given frequency for a portion of the area of the device. In comparison, the targeted QBIC is resonant across the entire device, at a single frequency [57].

Finally, we demonstrate that thermal metasurfaces are capable of preferentially emitting quasimonochromatic light with arbitrary SAM and OAM. Schematically envisioned in Fig. 9(a), such a thermal metasurface is designed to preferentially absorb an azimuthally varying phase profile. Figures 9(b) and 9(c) map the required inplane orientation angles of the local and nonlocal elements of the device aimed at producing the OAM beam mapped in Figs. 9(d) and 9(e). Note that an alternate device geometry is used for this demonstration due to its improved performance; the details of the geometry of this device are given in Appendix F along with a brief discussion of other possible thermal metasurface implementations following the basic requirements outlined above. To study the emitted light of such a device, we record the absorption due to excitation by normally incident OAM beams of varying OAM integer $\ell$. That is, we calculated the absorption due to excitation by waves

$$
\begin{aligned}
E(x, y)= & A_{0}\left(x^{2}+y^{2}\right)^{|\ell| / 2} \exp \left[-\left(x^{2}+y^{2}\right) / w_{0}^{2}\right] \\
& \times \exp [i 2 \ell \operatorname{atan} 2(y, x)]
\end{aligned}
$$

where $w_{0}$ is the beam waist and $A_{0}$ determines the field strength. Since the OAM basis is complete, a simulation for all values of $\ell$ exhaustively characterizes the emission of the device at normal incidence. However, a completely exhaustive demonstration would require an infinite number of simulations spanning both all values of $\ell$ and all incident angles. We limit the demonstration to normal incidence and test values of $\ell$ near the designed value. Shown in Fig. 9(f), we see that, at the band-edge frequency, the designed OAM is strongly absorbed, but for other values of $\ell$ a weaker and spectrally shifted absorption is observed. Consequently, at the band-edge frequency, light with net OAM is emitted, with efficiencies to each order $\ell$ depicted in Fig. $9(\mathrm{~g})$.

We note that the pattern in Fig. 9(f) strongly resembles the band structure of the underlying QBIC, seen in Fig. 7, for instance. Since $\ell$ determines the integer number of $2 \pi$ that the incoming phase is azimuthally wrapped, it tracks the direction and magnitude of the incoming angle at a 


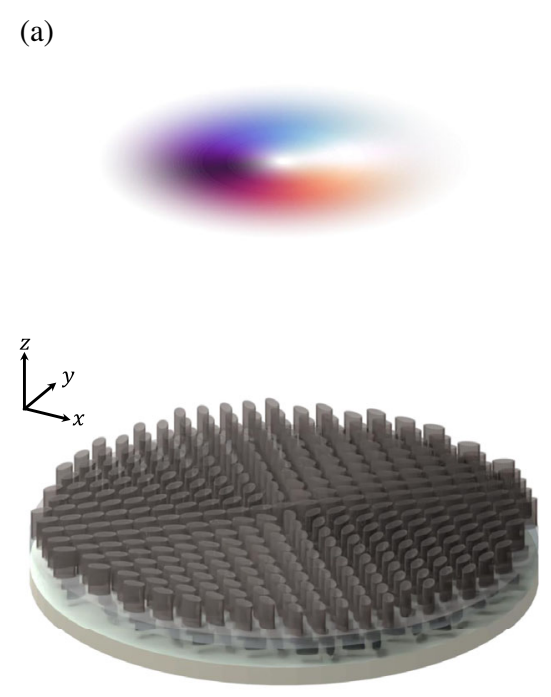

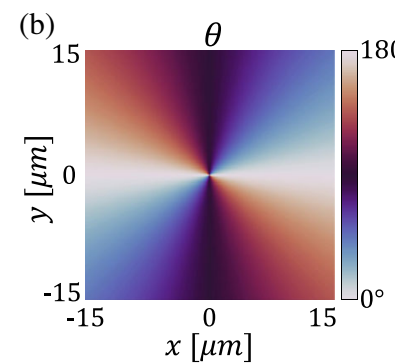

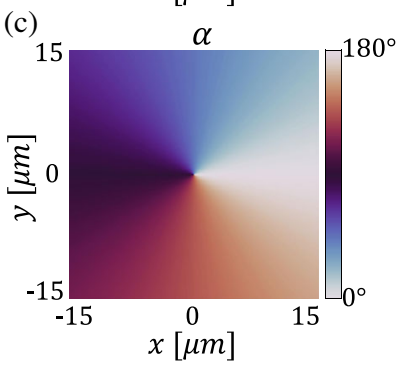

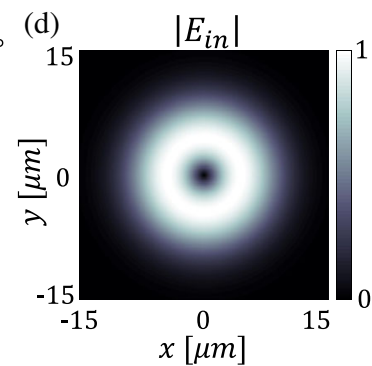

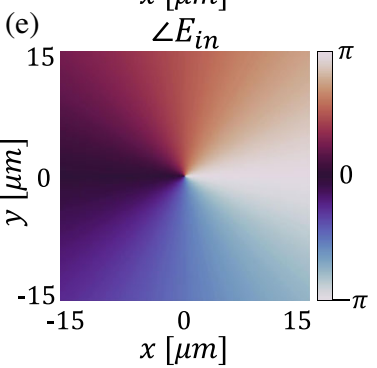

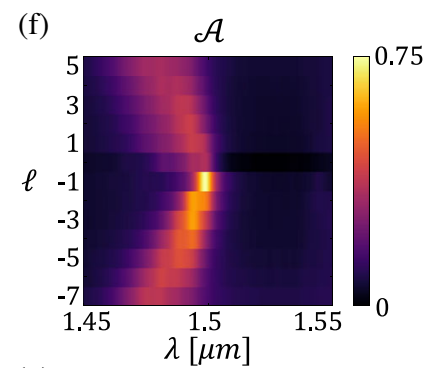

(g)

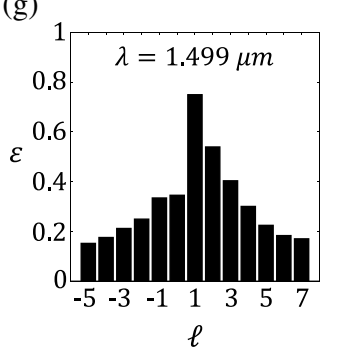

FIG. 9. Thermal metasurface generating quasimonochromatic SAM or OAM light. (a) Schematic of a thermal metasurface with an azimuthally varying directivity. (b),(c) Angular parameters defining a $30 \times 30 \mu \mathrm{m}$ thermal metasurface generating RCP light with an OAM of $\ell=1$ at $\lambda=1.5 \mu \mathrm{m}$. (d),(e) Example electric field profile of LCP light launched in full-wave simulations to study the absorption of normally incident OAM light. (f) Absorption spectra for various OAM beams such as that depicted in (d) and (e). (g) Emissivity into the OAM channels at the peak wavelength, $\lambda=1.499 \mu \mathrm{m}$, showing preferential emission to the designed OAM order and emission with overall net OAM.

given point across the device. A correspondence between the linear and azimuthal phase gradients is, therefore, expected: Just as a thermal metasurface with a linear phase gradient shifts the band structure so that the band-edge mode emits to a nonzero angle, a thermal metasurface with an azimuthal phase gradient shifts the preferred emissivity to a nonzero $\ell$. The exhaustive demonstration of this equivalent physics for linear momentum shifts in Fig. 7, therefore, supplies strong evidence that the limited set of simulations in Fig. 9 is indeed representative of the emission of the device.

Finally, we note that the purity of the generated OAM in Fig. 9 is limited primarily by practical considerations, not fundamental ones. As discussed before and demonstrated in Fig. 7, the angular range of emission for a thermal metasurface is related to the $Q$ factor and the band curvature. Increasing the $Q$ factor and sharpness of the band increases the distance the QBIC travels in plane before scattering, and, therefore, it increases the spatial coherence and effective aperture size, which narrows the angular range of emission. For an azimuthally wrapped phase gradient, narrowing the angular range emitted at each location along the device increases the OAM purity. However, the energy contained in the QBIC must be localized within the bounds of the device, or it simply escapes in plane and does not contribute to the emission. Therefore, a compact device requires a flat band and low $Q$ factor, which limits the OAM purity. Since a compact device is a practical requirement for the numerical demonstration by full-wave simulations, the result has somewhat limited purity. However, an experimental platform aiming at high-purity OAM may largely improve on this result by increasing the size of the device, increasing the $Q$ factor, and employing a QBIC with a sharper band. A QBIC with an isotropic angular dispersion is also desirable. Despite these limitations, net OAM and pure SAM are generated by our thermal metasurface platform constructed out of purely rational design principles.

TABLE I. Complete control of thermal emission.

\begin{tabular}{lccc}
\hline \hline Property & TCMT parameters & Control mechanism & Metasurface parameters \\
\hline$A$ & $\sqrt{1-|r|^{2}}, \gamma_{i}$ & Critical coupling & $W_{d}, L_{d}, \kappa$ \\
$\Phi$ & $\Phi_{c}$ & Local scattering & $W_{m}, L_{m}, \theta$ \\
$\chi$ & $\beta=\theta-\phi$ & Chiral perturbation + local birefringence & $W_{m}, L_{m}, \theta, \alpha$ \\
$\psi$ & $\theta$ & Chiral perturbation + local birefringence & $W_{m}, L_{m}, \theta, \alpha$ \\
$\tau_{c}$ & Perturbation & $W_{d}, L_{d}$ \\
$L_{c}$ & $\tau=1 / \gamma_{r}$ & Perturbation + band curvature & $W_{d}, L_{d}, b$ \\
$p$ & $\sqrt{b \tau}$ & Multiplexing & Not applicable \\
\hline \hline
\end{tabular}


Computational approaches, such as inverse design [73], may be employed to further optimize the performance of this as well as the previous designs. We, therefore, consider thermal metasurfaces to be a uniquely promising platform for ultracompact thermal light generation with arbitrary SAM and OAM.

\section{CONCLUSION}

In this work, we have introduced a general platform for thermal metasurfaces building off the recent and rapid development of nonlocal metasurfaces (see Appendix G). In our view, the thermal metasurfaces introduced in this work represent the completion of the program of compactification to construct designer quasimonochromatic electromagnetic waves introduced by optical metasurfaces (Fig. 1). In contrast to conventional coherent metasurfaces, thermal metasurfaces produce partially coherent wave fronts described by the electric cross-spectral density, characterized by the parameters $A, \Phi, \chi, \psi, \tau_{c}, L_{c}$, and $p$ (Fig. 2). Table I summarizes how each parameter may be controlled by the geometry and symmetries in our thermal metasurface. To this end, we introduced a dual perturbative framework that enables the simultaneous control of the radiative and nonradiative lifetimes of a QBIC via geometric and material perturbation, respectively (Fig. 3). Together, these degrees of freedom enable control over both the temporal coherence $\tau_{c}$ and the amplitude of emitted light, $A$ (Appendix C and Fig. 10). We showed further that the radiative lifetime along with the band curvature of the QBIC, characterized by the Taylor coefficient $b$, determines the coherence length $L_{c}=\sqrt{b \tau}$ (Fig. 4), tunable separately from the temporal coherence by band structure engineering (see also Appendixes D and E).

Next, we developed a temporal coupled mode theory providing a framework to study the fundamental limitations in controlling the polarization state and directionality of thermal emission, constrained, in particular, by time-reversal symmetry and reciprocity. The key lesson of this general theory is that a thermal metasurface over a mirror plane must engineer both local and nonlocal responses simultaneously (Fig. 5). Guided by this result, we proposed an example geometry composed of a structured slab of doped silicon that controls the local response via a monatomic array of elliptical pillars while simultaneously supporting a symmetry-protected BIC that is perturbed into a QBIC by a dimer array placed at a distinct plane. This geometry is capable of emitting any polarization state to normal incidence (Fig. 6). For the special case of circular polarization, the geometric phase allows a particularly convenient method of varying the local phase with little change of the resonant wavelength of the QBIC. Leveraging this tool, we demonstrated thermal metasurfaces with unidirectional emission of a single chosen spin (Fig. 7), metasurfaces focusing thermal emission to a desired far-field position (Fig. 8), and metasurfaces capable of producing designer spin and orbital angular momentum (Fig. 9). These capabilities are extendable to any desired polarization state, or, indeed, vectoral wave fronts whose polarization state varies spatially, by varying the propagation phase while adjusting the resonant frequency of the QBIC accordingly (Appendix A). Lastly, while in most applications polarized light is generally preferable, we note that the degree of polarization $p$ may be also controlled if desired by multiplexing this approach: By suitably arraying patches of mixed polarization, the net polarization in the far field may be partially unpolarized.

In conclusion, we emphasize that the framework developed here leverages simultaneously the recent advances in local and nonlocal metasurface physics in order to expand our command of light directly at the point of origin. Thermal metasurfaces offer a miniaturized designer optical source fabricable by mature micro- and nanofabrication technologies and well-established design principles. The resulting devices are capable of direct thermal generation of structured light without the optical losses conventionally required by successive filters. These principles may apply also to manipulating LED emission and photoluminescence, and future work may incorporate narrow-band dopants for additional control over the resulting spectrum. Last, we note that the control over local and nonlocal scattering phenomena enabled by this platform may be incorporated into systems with nonlinearity and/or optical gain for novel control and generation of coherent light. We, therefore, view this platform as the next step of a program enabling hybrid local-nonlocal metasurfaces with unprecedented multifunctionality, offering an attractive approach for compact and high-performance next-generational classical and quantum optics.

\section{ACKNOWLEDGMENTS}

The authors thank Nanfang Yu, Stephanie Malek, and Yarden Mazor for useful discussions regarding nonlocal metasurfaces. This work was supported by the Department of Defense Vannevar Bush Faculty Fellowship, the Office of Naval Research with Grant No. N00014-19-1-2011, and the Air Force Office of Scientific Research MURI program. S. A. M. acknowledges support from a Rubicon fellowship from the Dutch Research Council (NWO).

\section{APPENDIX A: GENERAL COUPLED MODE THEORY}

We provide the general coupled mode theory solution for the device class described in Fig. 7, whose local response is a half wave plate but with arbitrary propagation phase (as demonstrated, for instance, in Ref. [9]). This result shows that the numerical demonstrations using circularly polarized light in the main text may be generalized to any polarization state.

We begin with the local background scattering matrix [Eq. (40)]: 


$$
C=\exp \left(i \Phi_{C}\right)\left[\begin{array}{cc}
\cos (2 \theta) & \sin (2 \theta) \\
\sin (2 \theta) & -\cos (2 \theta)
\end{array}\right]
$$

As described in the main text, we find a solution to this nonlocal scattering problem in the form

$$
|d\rangle=\sqrt{\frac{2}{\tau}} \frac{1+i}{2}\left[\begin{array}{c}
\cos (\phi)+i \cos (2 \theta-\phi) \\
\sin (\phi)+i \sin (2 \theta-\phi)
\end{array}\right] e^{i \Phi_{c} / 2},
$$

which may be verified to satisfy Eqs. (26) and (27). The resulting scattering matrix is

$$
S=e^{i \Phi_{c}}\left(C_{0}+\frac{1}{\Omega \tau-i} D_{0}\right)
$$

where

$$
C_{0}=\left[\begin{array}{cc}
\cos (2 \theta) & \sin (2 \theta) \\
\sin (2 \theta) & -\cos (2 \theta)
\end{array}\right]
$$

$$
D_{0}=\left[\begin{array}{cc}
{[\cos (\theta+\beta)+i \cos (\theta-\beta)]^{2}} & \cos (2 \theta) \sin (2 \beta)+i \sin (2 \theta) \\
\cos (2 \theta) \sin (2 \beta)+i \sin (2 \theta) & {[\sin (\theta+\beta)+i \sin (\theta-\beta)]^{2}}
\end{array}\right],
$$

$$
\Omega=\omega-\omega_{0}-i \gamma_{i}
$$

and $\beta=\theta-\phi$.

We are interested, in particular, when this symmetric scattering matrix is singular, in which case it has at least one eigenvalue of 0 , indicating perfect absorption of the corresponding eigenvector. The determinant of $S$ is

$$
\operatorname{Det}[S]=-e^{i 2 \Phi_{c}} \frac{\Omega \tau+i}{\Omega \tau-i},
$$

which vanishes only if

$$
\Omega \tau=-i
$$

Since $\Omega$ is the difference of the excitation frequency and the complex QBIC frequency, this condition constrains both the real and imaginary parts and means that perfect absorption happens only at the critically coupled condition:

$$
\begin{gathered}
\omega=\omega_{0}, \\
\gamma_{i} \tau=1 .
\end{gathered}
$$

In this case, we may find the eigenvector of $S$ with the zero eigenvalue:

$$
\left|e^{*}\right\rangle=\left[\begin{array}{cc}
\cos (\theta) & -\sin (\theta) \\
\sin (\theta) & \cos (\theta)
\end{array}\right]\left[\begin{array}{c}
\cos (\beta) \\
i \sin (\beta)
\end{array}\right],
$$

which is the characteristic polarization state emitted or absorbed by the device. With this form, we may now detune from the critically coupled condition and write the reflection coefficient

$$
r_{e e}(\omega)=\left\langle e|S| e^{*}\right\rangle=e^{i \Phi_{c}} \frac{\Omega \tau+i}{\Omega \tau-i}
$$

which we may write in the more suggestive form

$$
r_{e e}(\omega)=e^{i \Phi_{c}}\left(1+\frac{2 i}{\left(\omega-\omega_{0}-i \gamma\right) \tau-i}\right),
$$

wherein the factor of unity is due to the local response (reflection of $\left|e^{*}\right\rangle$ to the state $|e\rangle$ ) and the remaining factor the nonlocal response (the Fano resonant absorption). We may then model the resonant frequency as a function of momentum with the replacement

$$
\omega_{0}-i \gamma_{i} \rightarrow \omega_{\text {res }}(k) .
$$

For QBICs folded into the continuum by a periodaltering perturbation, the dipole moment of the perturbative scattering dominates, meaning that the radiative loss rate $\gamma$ is approximately independent of $k$ [62]. The real part follows the band structure of the mode, which we may Taylor expand about $k=0$, yielding

$$
\omega_{\text {res }}(k) \approx \omega_{0}+\frac{b}{2} k^{2}-i \gamma_{i} .
$$

We then write the Fresnel coefficient in frequency momentum coordinates as

$r_{e e}(k, \omega)=e^{i \Phi_{c}}\left(1+\frac{2 i}{\left(\omega-\omega_{0}-\frac{b}{2} k^{2}-i \gamma_{i}\right) \tau-i}\right)$.

This result shows that we may extend control over the band-edge mode shown in the main text for circular polarization to any polarization state.

\section{APPENDIX B: DERIVATION OF THE DEPENDENCE OF THE NONRADIATIVE $Q$ FACTOR ON $\kappa$}

We briefly present a derivation that the nonradiative $Q$ factor should follow $Q_{l} \propto 1 / \kappa$ as demonstrated numerically 
in Fig. 3 of the main text. For a cavity with known electric field profile $E(\mathbf{r})$ and permittivity profile $\epsilon(\mathbf{r})$, and assuming equal energy is stored in the magnetic and electric fields, the nonradiative $Q$ factor has the form [74]

$$
Q_{l} \approx \frac{\iiint d \mathbf{r}^{3} \epsilon^{\prime}(\mathbf{r})|E(\mathbf{r})|^{2}}{\iiint d \mathbf{r}^{3} \epsilon^{\prime \prime}(\mathbf{r})|E(\mathbf{r})|^{2}}
$$

where the perturbed permittivity profile is $\epsilon(\mathbf{r})=\epsilon^{\prime}(\mathbf{r})+$ $i \epsilon^{\prime \prime}(\mathbf{r})$. We may evaluate Eq. (B1) in terms of the fraction of stored energy in air, silicon, and the filler material, which, for our example here, have refractive indices $\epsilon_{\text {air }}=1$, $\epsilon_{\mathrm{si}}=(n+i \kappa)^{2}=(3.45+i \kappa)^{2}$, and $\epsilon_{f}=1.4^{2}$, respectively. The fraction of stored energy in silicon is called $f_{1}$, and the fraction in the filler material is called $f_{2}$, making the fraction in air $1-f_{1}-f_{2}$. In this case, Eq. (B1) becomes

$$
Q_{l} \approx \frac{f_{1}\left(n^{2}-\kappa^{2}\right)+f_{2} \epsilon_{f}+\left(1-f_{1}-f_{2}\right) \epsilon_{\mathrm{air}}}{2 n f_{1}} \frac{1}{\kappa},
$$

where we use

$$
\epsilon^{\prime \prime}=2 n \kappa .
$$

Or, since $\kappa \ll n$,

$$
Q_{l} \approx \frac{f_{1} n^{2}+f_{2} \epsilon_{f}+\left(1-f_{1}-f_{2}\right) \epsilon_{\mathrm{air}}}{\kappa} \frac{1}{\kappa} .
$$

Equation (B4) agrees with the form $Q_{l}=\kappa_{0} / \kappa$ observed in Fig. 3(d) and gives the constant of proportionality to be

$$
\kappa_{0}=\frac{f_{1} n^{2}+f_{2} \epsilon_{f}+(1-f) \epsilon_{\mathrm{air}}}{2 n f} .
$$

Retrieving $f_{1}=0.2649$ and $f_{2}=0.2626$ from the fullwave simulations in the case that $\kappa=10^{-2.84}$, we calculate the value $\kappa_{0}=2.27$ from Eq. (B5), in reasonable agreement with the value $\kappa_{0}=2.22$ fitting the data in Fig. 3(d).

\section{APPENDIX C: CONTROL OF EMISSIVITY AND LIFETIME}

Here, we discuss implementations of a metasurface library enabling large control over the emissivity and lifetime. Figure 10(a) shows an implementation based on Fig. 3 with perturbations controlling the radiative and nonradiative lifetimes, where $Q_{r}$ varies according to the geometric perturbation and $Q_{l}$ according to the dopant concentration. Equations (12) and (13) are visualized in this parameter space in Figs. 10(b) and 10(c), respectively, highlighting how the dual perturbation offers arbitrary control over linewidth and peak emissivity of the band-edge mode. Given the maturity of micro- and nanofabrication platforms, these values may be spatially varied across the surface of a device by spatially varying the geometric perturbation and dopant concentration as desired. This variation may be achieved via successive masking and implantation steps or potentially gray-scale lithography to yield spatially varying concentration and diffusion depth of the dopants.

Alternatively, we note that a lossless material such as intrinsic silicon may be used to create the metaunits, while a thin layer of a second, lossy material may be deposited, any where in the near field of the device (e.g., indium tin oxide for its widely tunable properties in the infrared [75]). Spatially patterning this second material would introduce a spatially varying loss. If the material is thin, the change to the resonant frequency of the mode is small; if required, this loss may be adjusted by changing the geometry of the lossless material.

Finally, we briefly address the effects due to intrinsic carriers in ostensibly lossless materials such as silicon. For increased signal strength, it is desirable to operate a thermal metasurface at elevated temperatures $T$. In any semiconductor, the intrinsic carrier concentration increases exponentially with temperature, according to [76]
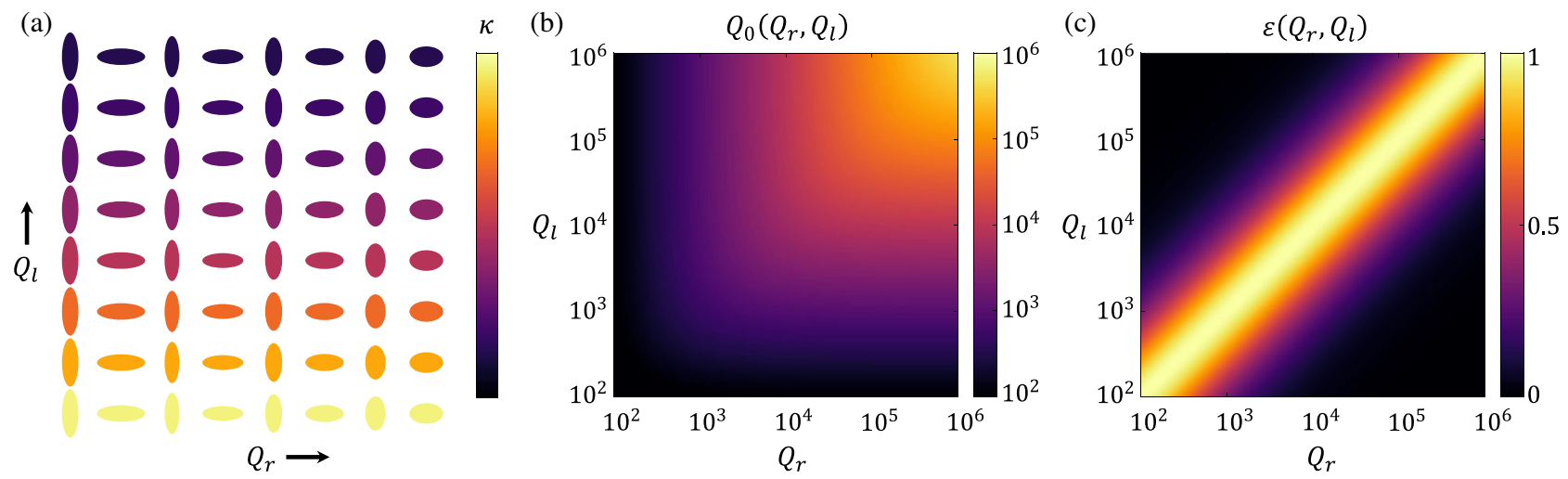

FIG. 10. Controlling spectral linewidth and peak emissivity with a doped photonic crystal slab. (a) Library of metaunits varying the dopant concentration (extinction coefficient $\kappa$ ) to control the nonradiative $Q$ factor $Q_{l} \propto 1 / \kappa$ and perturbation magnitude $\delta$ to control the radiative $Q$ factor $Q_{r} \propto 1 / \delta^{2}$ for $x$-polarized light. (b) Fano resonance $Q$ factor $Q_{0}$ as a function of $Q_{l}$ and $Q_{r}$. (c) Peak emissivity of the quasimonochromatic thermal source, as a function of $Q_{l}$ and $Q_{r}$. 


$$
n_{i}(T)=n_{\mathrm{RT}} \exp \left[-\frac{E_{g}}{2 k_{B}}\left(\frac{1}{T}-\frac{1}{T_{\mathrm{RT}}}\right)\right],
$$

where $n_{\mathrm{RT}}=9.65 \times 10^{9} \mathrm{~cm}^{-3}$ is the intrinsic carrier concentration of silicon at room temperature, $T_{\mathrm{RT}}=300 \mathrm{~K}$, $E_{g}=1.12 \mathrm{eV}$ is the band gap of silicon, and $k_{B}$ is Boltzmann's constant. According to Ref. [77], the values of loss in the main text $\left(\kappa \approx 10^{-3}\right)$ are achieved at $\lambda_{0}=$ $1.55 \mu \mathrm{m}$ near carrier concentrations on the order of $10^{18} \mathrm{~cm}^{-3}$, which means at room temperature the effect of intrinsic carriers is completely negligible. However, the intrinsic carrier concentration becomes comparable to $10^{18} \mathrm{~cm}^{-3}$ for silicon at around $T \approx 1100 \mathrm{~K}$ [76]. In this regard, in place of doping, the intrinsic carriers at high temperatures may be considered as controllable optical loss, albeit in a uniform manner across the device. Alternatively, in devices with design dopant profiles, they must operate below a certain temperature (here, roughly $T=1100 \mathrm{~K}$ ).

\section{APPENDIX D: DERIVATION OF EQ. (19)}

Here, we carry out the derivation of the spectral degree of coherence of the QBIC metasurface. We assume that our thermal metasurface may be modeled using (i) the Schell model and (ii) quasihomogenous planar secondary sources [2]. The first assumption implies that the spectral degree of coherence is shift invariant, having the form $\mu\left(\boldsymbol{\rho}_{2}-\boldsymbol{\rho}_{1}, \omega\right)$, while the second assumption means that the spectral intensity spatially varies slowly compared to $\mu\left(\boldsymbol{\rho}_{2}-\boldsymbol{\rho}_{1}, \omega\right)$. We note that not all imaginable thermal metasurfaces satisfy these assumptions, since they require spatially constant temporal coherence and (as we establish below) band curvature. However, these often-used assumptions cover many cases of interest (including the examples herein), and the insights afforded by the simplicity of these assumptions may guide future work where, for instance, the radiative lifetime and/or in-plane Bragg scattering varies across the device. Under these basic assumptions, it can be shown [2] that the radiant intensity of light emitted to an angle $\theta_{x}$ in the far field is related to the spatial Fourier transform of the spectral degree of coherence at the source plane, namely,

$$
J_{\omega}\left(\theta_{x}\right)=\left(2 \pi k_{0}\right)^{2} A_{0} I_{0}(\omega) \mu\left(k_{0} s_{x}, \omega\right) \cos ^{2}\left(\theta_{x}\right),
$$

where $A_{0}$ is the area of the source emitting with a positionindependent spectral density $I_{0}(\omega)$ and $k_{0} s_{x}=k_{0} \sin \left(\theta_{x}\right)$ is the spatial frequency associated with the plane wave emitted toward $\theta_{x}$.
Meanwhile, the intensity emitted to angle $\theta_{x}$ by a body with emissivity $\varepsilon\left(\theta_{x}, \omega\right)$ is

$$
J_{\omega}\left(\theta_{x}\right)=I_{\mathrm{BB}}(\omega, T) \varepsilon\left(\theta_{x}, \omega\right),
$$

where $I_{\mathrm{BB}}(\omega, T)$ is the blackbody spectral intensity. Hence, from Eqs. (D1) and (D2), we obtain the spectral degree of coherence in the space-frequency domain, which has the form (see Ref. [2])

$$
\mu(x, \omega)=\frac{F(x, \omega)}{F(0, \omega)},
$$

where in our case

$$
F(x, \omega)=\int \frac{\varepsilon\left(k_{0} s_{x}, \omega\right)}{1-s_{x}^{2}} e^{i k_{0} s_{x} x} d\left(k_{0} s_{x}\right) .
$$

For simplicity, we now constrain our study to near normal incidence, such that the inclination factor is $1-s_{x}^{2} \approx 1$, and we assume the local response to be lossless, i.e., $r_{0}=1$ in Eq. (15). In this case, using the thermal emissivity expression in Eq. (18), Eq. (D4) yields

$$
\mu(x, \omega)=\frac{1}{\operatorname{Im}[\xi(\omega)]} \operatorname{Im}\left[\xi(\omega) \exp \left(-\frac{|x|}{\xi(\omega)}\right)\right],
$$

where

$$
\xi(\omega)=\sqrt{\frac{b \tau / 2}{i-\left(\omega-\omega_{0}\right) \tau}} .
$$

\section{APPENDIX E: CORRELATION LENGTH FROM THE FLUCTUATION-DISSIPATION THEOREM}

The cross-spectral density tensor for vector fields is given by Eq. (9), written as an outer product:

$$
\mathbf{W}\left(\mathbf{r}_{1}, \mathbf{r}_{2}, \omega\right)=\left\langle\mathbf{E}\left(\mathbf{r}_{1}, \omega\right) \mathbf{E}\left(\mathbf{r}_{2}, \omega\right)^{\dagger}\right\rangle .
$$

As the field arises due to thermal sources inside the medium, we have for the fields

$$
\mathbf{E}(\mathbf{r})=-i \omega \mu_{0} \iiint_{V} \overline{\overline{\mathbf{G}}}\left(\mathbf{r}, \mathbf{r}^{\prime}\right) \cdot \mathbf{J}\left(\mathbf{r}^{\prime}\right) d^{3} \mathbf{r}^{\prime},
$$

where $\overline{\overline{\mathbf{G}}}\left(\mathbf{r}, \mathbf{r}^{\prime}\right)$ is the Green's tensor, $\mathbf{J}\left(\mathbf{r}^{\prime}\right)$ is a thermal current density, and the integral is over the source volume. The cross-spectral density tensor, thus, becomes

$$
\mathbf{W}\left(\mathbf{r}_{1}, \mathbf{r}_{2}, \omega\right)=\omega^{2} \mu_{0} \iiint_{V} d^{3} \mathbf{r}^{\prime} \iiint_{V} d^{3} \mathbf{r}^{\prime \prime}\left\langle\left[\overline{\bar{G}}\left(\mathbf{r}_{1}, \mathbf{r}^{\prime}\right) \cdot \mathbf{J}\left(\mathbf{r}^{\prime}\right)\right] \cdot\left[\overline{\bar{G}}\left(\mathbf{r}_{2}, \mathbf{r}^{\prime \prime}\right) \cdot \mathbf{J}\left(\mathbf{r}^{\prime \prime}\right)\right]^{\dagger}\right\rangle,
$$

which can conveniently be rewritten as 


$$
\mathbf{W}\left(\mathbf{r}_{1}, \mathbf{r}_{2}, \omega\right)=\omega^{2} \mu_{0} \iiint_{V} d^{3} \mathbf{r}^{\prime} \iiint_{V} d^{3} \mathbf{r}^{\prime \prime} \overline{\overline{\mathbf{G}}}\left(\mathbf{r}_{1}, \mathbf{r}^{\prime}\right)\left\langle\mathbf{J}\left(\mathbf{r}^{\prime}\right) \cdot \mathbf{J}^{\dagger}\left(\mathbf{r}^{\prime \prime}\right)\right\rangle \overline{\overline{\mathbf{G}}}^{\dagger}\left(\mathbf{r}_{2}, \mathbf{r}^{\prime \prime}\right) .
$$

For thermal processes, the correlation of the stochastic current densities is known through the fluctuation-dissipation theorem [78]:

$$
\left\langle J_{k}\left(\mathbf{r}^{\prime}, \omega^{\prime}\right) J_{l}^{*}\left(\mathbf{r}^{\prime \prime}, \omega^{\prime \prime}\right)\right\rangle=\frac{\omega \Theta(\omega, T)}{\pi} \operatorname{Im}(\varepsilon) \delta_{k l} \delta\left(\mathbf{r}^{\prime}-\mathbf{r}^{\prime \prime}\right) \delta\left(\omega^{\prime}-\omega^{\prime \prime}\right),
$$

where $\Theta(\omega, T)=\hbar \omega /\left[\exp \left(\hbar \omega / k_{B} T\right)-1\right], \hbar$ is the reduced Planck's constant, and $k_{B}$ is Boltzmann's constant. Using this correlation, we can write

$$
\mathbf{W}\left(\mathbf{r}_{1}, \mathbf{r}_{2}, \omega\right)=\frac{\omega^{3} \mu_{0}^{2} \Theta(\omega, T)}{\pi} \operatorname{Im}(\varepsilon) \iiint_{V} d^{3} \mathbf{r}^{\prime} \iiint_{V} d^{3} \mathbf{r}^{\prime \prime} \overline{\bar{G}}\left(\mathbf{r}_{1}, \mathbf{r}^{\prime}\right) \cdot \overline{\bar{G}}^{\dagger}\left(\mathbf{r}_{2}, \mathbf{r}^{\prime \prime}\right) \delta\left(\mathbf{r}^{\prime}-\mathbf{r}^{\prime \prime}\right) .
$$

Finally, evaluating one of the integrals using the delta function:

$$
\mathbf{W}\left(\mathbf{r}_{1}, \mathbf{r}_{2}, \omega\right)=\frac{\omega^{3} \mu_{0}^{2} \Theta(\omega, T)}{\pi} \operatorname{Im}(\varepsilon) \iiint_{V} d^{3} \mathbf{r}^{\prime} \overline{\overline{\mathbf{G}}}\left(\mathbf{r}_{1}, \mathbf{r}^{\prime}\right) \cdot \overline{\bar{G}}^{\dagger}\left(\mathbf{r}_{2}, \mathbf{r}^{\prime}\right) .
$$

Evaluating this integral further requires knowledge of the Green's tensor of the structure under consideration. In this work, we focus on metasurfaces where the response is dominated by a single resonant mode. Assuming that the quality factors are high, we may therefore approximate the Green's tensor as [79]

$$
\begin{aligned}
\overline{\overline{\mathbf{G}}}\left(\mathbf{r}, \mathbf{r}^{\prime}\right) & =\frac{1}{2 i \omega \mu_{0}} \sum_{n=1}^{\infty} \mathbf{e}_{n}(\mathbf{r}) \otimes \mathbf{e}_{n}^{\mathrm{T}}\left(\mathbf{r}^{\prime}\right) \\
& \approx \frac{1}{2 i \omega \mu_{0}} \mathbf{e}_{1}(\mathbf{r}) \otimes \mathbf{e}_{1}^{\mathrm{T}}\left(\mathbf{r}^{\prime}\right),
\end{aligned}
$$

valid for $\mathbf{r} \neq \mathbf{r}^{\prime}$ and where we take $\mathbf{e}_{1}$ to be the field profile of the resonant mode, which from here on we simply refer to as e. Using the identity $\overline{\bar{G}}\left(\mathbf{r}^{\prime}, \mathbf{r}^{\prime \prime}\right)=\bar{G}^{\mathrm{T}}\left(\mathbf{r}^{\prime \prime}, \mathbf{r}^{\prime}\right)$, we can thus write the volume integral over the Green's functions in Eq. (E7) as

$$
\begin{aligned}
& \iiint_{V} d^{3} \mathbf{r}^{\prime} \overline{\overline{\mathbf{G}}}\left(\mathbf{r}_{1}, \mathbf{r}^{\prime}\right) \cdot \overline{\bar{G}}^{\dagger}\left(\mathbf{r}_{2}, \mathbf{r}^{\prime}\right) \\
& \quad=\iiint_{V} d^{3} \mathbf{r}^{\prime} \overline{\bar{G}}\left(\mathbf{r}_{1}, \mathbf{r}^{\prime}\right) \cdot \overline{\mathbf{G}}^{*}\left(\mathbf{r}^{\prime}, \mathbf{r}_{2}\right) \\
& \quad=-\frac{\mathbf{e}\left(\mathbf{r}_{1}\right) \otimes \mathbf{e}^{\dagger}\left(\mathbf{r}_{2}\right)}{4 \omega^{2} \mu_{0}^{2}} \iiint_{V} d^{3} \mathbf{r}^{\prime} \mathbf{e}^{\mathrm{T}}\left(\mathbf{r}^{\prime}\right) \cdot \mathbf{e}^{*}\left(\mathbf{r}^{\prime}\right) .
\end{aligned}
$$

Given that the system is lossy, the integral of the field profile norm over the source volume converges to a finite number, which we refer to as $\zeta$ in the following.

Inserting Eq. (E9) into the expression for the crossspectral density tensor, we find

$$
\mathbf{W}\left(\mathbf{r}_{1}, \mathbf{r}_{2}, \omega\right)=-\frac{\omega \Theta(\omega, T) \operatorname{Im}(\varepsilon) \zeta}{4 \pi} \mathbf{e}\left(\mathbf{r}_{1}\right) \otimes \mathbf{e}^{\dagger}\left(\mathbf{r}_{2}\right)
$$

Given that the metasurface consists of a periodic medium, the field profile is given by $\mathbf{e}(\mathbf{r}, \mathbf{k})=\mathbf{u}(\mathbf{r}, \mathbf{k}) e^{i \mathbf{k} \cdot x}$, where $\mathbf{u}(\mathbf{r}, \mathbf{k})$ is a periodic function that is multiplied by the phase propagator to obtain the field profile anywhere. Focusing on the cross-spectral density along the $\hat{x}$ direction with $x_{2}>x_{1}$ (without loss of generality), we obtain

$$
\begin{aligned}
& \mathbf{W}\left(\mathbf{r}_{1}, \mathbf{r}_{2}, \omega\right) \\
& =-\frac{\omega \Theta(\omega, T) \operatorname{Im}(\varepsilon) \zeta}{4 \pi}\left(\mathbf{u}\left(\mathbf{r}_{1}, \mathbf{k}_{\mathbf{x}}\right) \otimes \mathbf{u}^{\dagger}\left(\mathbf{r}_{2}, \mathbf{k}_{x}\right)\right) e^{i k_{x}\left(x_{2}-x_{1}\right)} .
\end{aligned}
$$

If we write the separation as $r=x_{2}-x_{1}$, we obtain for the magnitude of the cross-spectral density (over distances significantly longer than the unit cell)

$$
|\mathbf{W}(r, \omega)| \propto e^{-k^{\prime \prime}{ }_{x}},
$$

where $k^{\prime \prime}{ }_{x}$ is the imaginary part of the propagation constant along the $\hat{x}$ direction.

To demonstrate that this result agrees with the results derived in the main text, we assume that the dispersion has the parabolic form (16), but including an imaginary part for the complex frequency due to material losses:

$$
\omega+i \gamma=\omega_{0}+\frac{b}{2} k_{x}^{2}
$$

For the wave number, this result yields $k_{x}=\sqrt{2 / b}$ $\sqrt{\left(\omega-\omega_{0}\right)-i \gamma}$. At the band edge, $\omega=\omega_{0}$, we obtain for the imaginary part of the wave number

$$
k_{x}^{\prime \prime}=\sqrt{\gamma / b} .
$$

Inserting this result in Eq. (E12), we find that the decay length is given by 

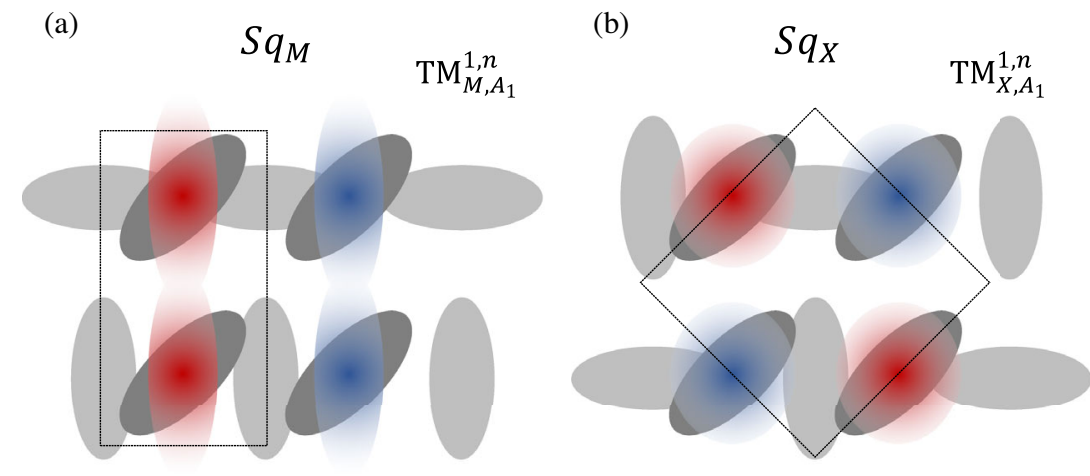

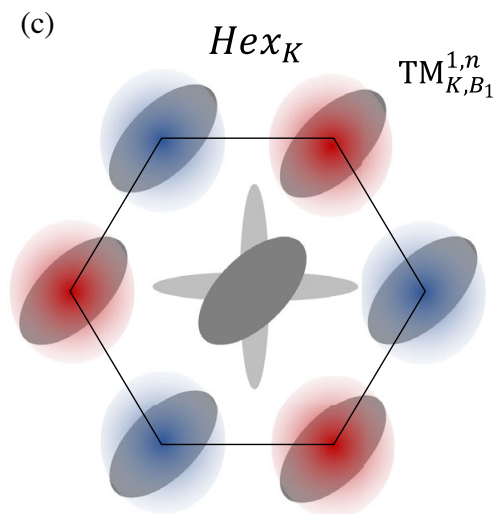

(d)
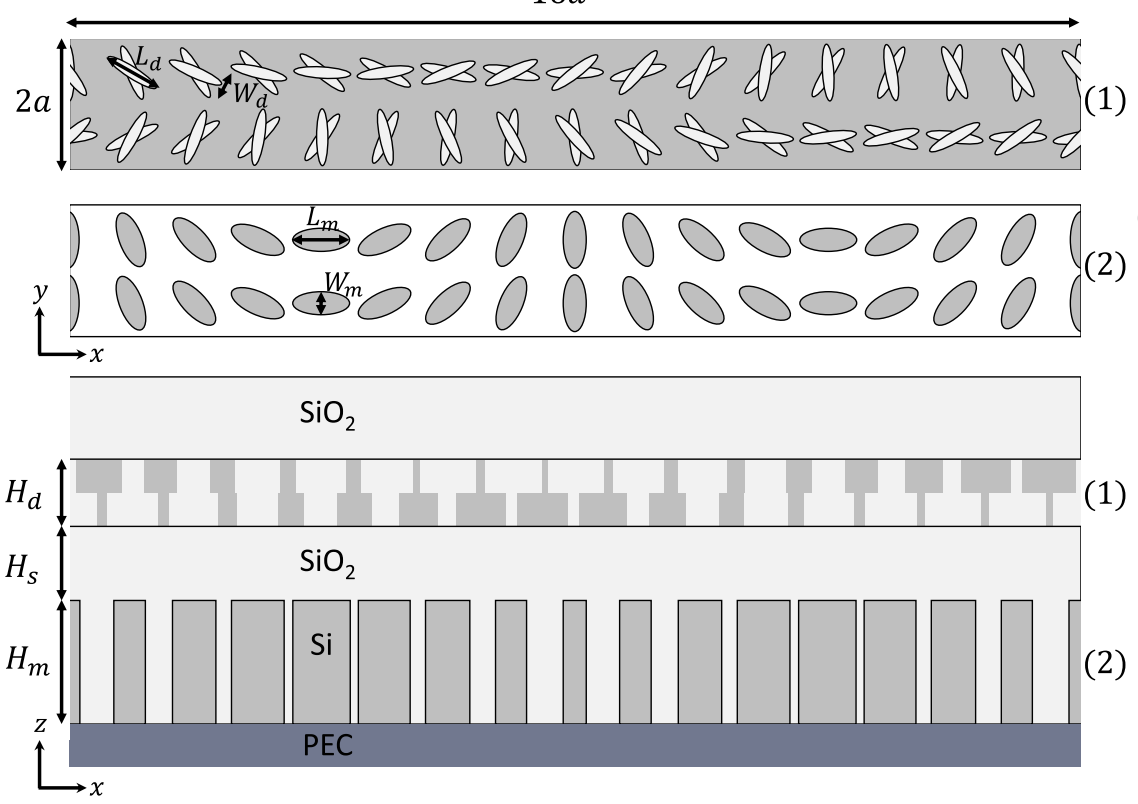

$(2)$

(f) 1.3

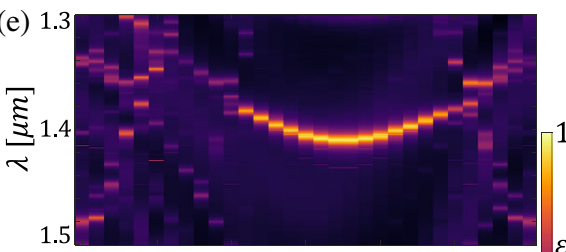

1
(1) $(\mathrm{g}$

(2)

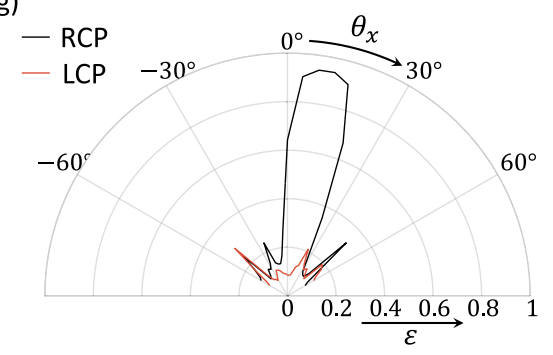

FIG. 11. Additional example geometries. (a)-(c) Three lattices controlling three different modes with both $Q$ factor and polarization angle control by varying the ellipticity and the in-plane orientation angles of the light-gray ellipses, respectively. The lattices and the targeted modes depicted are named according to the conventions in Ref. [54]. (d) An alternative configuration in the out-of-plane direction, having two elements: Element (1) is a dimerized PCS supporting a chiral QBIC, and element (2) is a monomer local metasurface atop a perfect electric conductor (PEC). (e),(f) RCP and LCP response for the device shown in (d). (g) Angular emissivity profile at the band-edge frequency, showing unidirectional emission of the designed spin.

$$
|\mathbf{W}(r, \omega)| \propto e^{-\sqrt{\gamma / b r}} .
$$

Hence, the $1 / e$ correlation length for the cross-spectral correlation density is $L_{c}=\sqrt{b / \gamma}=\sqrt{b \tau}$, in agreement with the results derived in the main text.

\section{APPENDIX F: EXAMPLE ALTERNATIVE GEOMETRIES}

We provide additional example geometries for potential use for thermal metasurfaces. We consider two categories of changes to the device in the main text: (i) a change of lattice and (ii) a change in out-of-plane positioning of the local and nonlocal elements. Figures 11(a)-11(c) depict lattices with $p 2$ space group having rectangular, square, and hexagonal symmetries, respectively [Fig. 11(a) is the lattice in the main text for comparison]. Overlaid are characteristic modes targeted by such a space group. Such alternatives may have the desirable characteristics of increasing isotropy of the band curvature.

Meanwhile, Fig. 11(d) shows an alternative configuration out of plane, wherein the local element is vertically separated from the nonlocal element. That is, a chiral QBIC structure that controls the nonlocal response (guided by Ref. [56]) is placed above a conventional local metasurface mirror (guided by Ref. [9]). If the spacer layer is sufficiently thick (as a rule of thumb, thicker than half the effective wavelength in the spacer material), the local and 
nonlocal elements operate independently, providing a more robust method of control over the local and nonlocal responses, at the cost of complexity and a minor increase in thickness (the entire stack is still on the order of the wavelength thick). This multilayer system is the one used in Fig. 9 to demonstrate OAM generation of light, and we note that devices based on multilayer fabrication have recently received substantial attention as a powerful method of increasing the command of light with metasurfaces (see, for instance, Ref. [80-82]).

We briefly discuss a few changes in the design principles of the device. In particular, we design the system with the understanding that the nonlocal element (1) scatters RCP light upward and downward, as demonstrated in Ref. [56]. Then, the downward scattered RCP state is anomalously reflected by the local element (2) before ultimately escaping upward. These two components of the scattered light interfere with each other, meaning that the local element directly controls the net scattering of the QBIC (consistent with the description in the main text that the nonlocal scattering is not independent of the local scattering). For instance, if the local element is ideally birefringent, the downward component reflects with preserved handedness (i.e., also as RCP light, but traveling upward). Its interference with the upward component is then constructive or destructive according to the round-trip phase accumulated by the downward component. When out of phase, destructive interference reduces the amplitude of the net RCP light scattering to free space; this reduction effectively translates to a reduction in radiative scattering rate (or an increase in $Q$ factor). Hence, both the thickness of the spacer layer as well as the geometric phase of the local element (2) impact the radiative $Q$ factor of the entire hybrid device. This control is duplicate to simply modifying the magnitude of the perturbation but must be kept in mind. For our design, we choose the geometric phase profile such that near-complete constructive interference occurs and adjust the loss in silicon to achieve critical coupling. Figures 11(e) and 11(f) show the emissivity for RCP and LCP light as a function of the angle and wavelength for the resulting structure, and Fig. 11(g) shows the angular profile of this system at the bandedge mode, showing RCP light emitting with a peak emissivity of 0.94 . The geometrical parameters of this device are $a=400 \mathrm{~nm}, H_{d}=500 \mathrm{~nm}, W_{d}=80 \mathrm{~nm}$, $L_{d}=340 \mathrm{~nm}, H_{m}=1000 \mathrm{~nm}, W_{m}=180 \mathrm{~nm}, L_{m}=360 \mathrm{~nm}$, and $H_{s}=600 \mathrm{~nm}$. The $i$ th monomer ellipse is rotated by $\theta_{i}=[(i-1) / 16] 360^{\circ}$, while the bottom set of dimer ellipses are oriented according to $\alpha_{i}=\theta_{i} / 2$ and $\alpha_{i}+90^{\circ}$. The corresponding dimer ellipses on top differ by angles $\Delta \alpha_{i}=56^{\circ}+10^{\circ} \cos \left(2 \alpha_{i}-30^{\circ}\right)$.

Lastly, we report the geometrical parameters used for the device in Fig. 8 in the main text (the device in Fig. 9 is identical but for the phase profile being that of a lens). The values are $a=300 \mathrm{~nm}, H_{d}=250 \mathrm{~nm}, W_{d}=$ $60 \mathrm{~nm}, L_{d}=240 \mathrm{~nm}, H_{m}=1000 \mathrm{~nm}, W_{m}=100 \mathrm{~nm}$,
$L_{m}=280 \mathrm{~nm}, H_{s}=100 \mathrm{~nm}, H_{f}=680 \mathrm{~nm}$, and $\kappa=0.01$. The $i$ th monomer ellipse is rotated by $\theta_{i}=[(i-1) /$ 16] $360^{\circ}$, and the $i$ th dimer ellipse is rotated by $\alpha_{i}=$ $\left(\theta_{i}-45^{\circ}\right) / 2$ (and its pair by $\alpha_{i}+90^{\circ}$ ).

\section{APPENDIX G: RECENT DEVELOPMENTS IN NONLOCAL METASURFACES}

Here, we briefly supply our perspective on how our work builds upon the ongoing study of nonlocality in metasurfaces: We view this thermal metasurface platform as a natural extension of recent and rapid advances in the field of nonlocal metasurfaces. Studied for many years, the role of symmetry in photonic crystals [83] was recently revived after the introduction of QBICs [58], clarifying that the optical lifetime of nonlocal phenomena in periodic structures may be robustly controlled within a simple rational design scheme. The concept of a QBIC was then extended from a scalar to a vectoral description, wherein the lifetime and eigenpolarization are governed by selection rules [54] and spin selectivity may be achieved by introduction of chiral perturbations [56]. By spatially varying the vectoral properties (eigenpolarization) but maintaining constant scalar properties (lifetime and resonant frequency), nonlocal metasurfaces shaping a wave front only at resonance were demonstrated $[53,55]$. This functionality is enabled by the excitation of spatially tailored dark modes, which extend the concept of the optical Fano resonance from the momentum-frequency domain to the space-frequency domain [57]. That is, in devices with high $Q$ factors and sharp bands, Fano resonances with spatially tailored dark modes are highly selective to the incoming wave front: Only the designed frequency, polarization, and phase profile will fully excite the QBIC. In this paper, by adding optical loss, we generalize this functionality to include wave-front-selective absorption and, by the fourth modal radiation law [60], wave-front-selective emission. To achieve unidirectional and complete absorption, a ground plane is added to the system, which (due to reciprocity and time-reversal symmetry) requires the extension of nonlocal metasurfaces to include simultaneous control over local and nonlocal responses. Future work will explore this expanded concept within lossless systems, wherein the broadband wave front is shaped locally and a narrow-band portion is independently shaped nonlocally. Furthermore, this system (i.e., the geometry in Fig. 7), in the absence of the mirror plane, is "two-faced," having a distinct eigenpolarization depending on the direction of excitation; that is, due to the combination of birefringence and dichroism, the system here may support nonorthogonal eigenpolarizations.

[1] M. Born and E. Wolf, Principles of Optics, 7th ed. (Cambridge University Press, Cambridge, England, 1999), ISBN: 978-0-521-64222-4. 
[2] E. Wolf, Introduction to the Theory of Coherence and Polarization of Light (Cambridge University Press, Cambridge, England, 2007), ISBN: 978-0-521-82211-4.

[3] E. Wolf, Correlation-Induced Changes in the Degree of Polarization, the Degree of Coherence, and the Spectrum of Random Electromagnetic Beams on Propagation, Opt. Lett. 28, 1078 (2003).

[4] N. Wiener, Generalized Harmonic Analysis, Acta Math. 55, 117 (1930).

[5] A. Khintchine, Korrelationstheorie der Stationaren Stochastischen Prozesse, Math. Ann. 109, 604 (1934).

[6] N. Yu, P. Genevet, M. A. Kats, F. Aieta, J.-P. Tetienne, F. Capasso, and Z. Gaburro, Light Propagation with Phase Discontinuities: Generalized Laws of Reflection and Refraction, Science 334, 333 (2011).

[7] N. Yu and F. Capasso, Flat Optics with Designer Metasurfaces, Nat. Mater. 13, 139 (2014).

[8] Y. Hu, X. Wang, X. Luo, X. Ou, L. Li, Y. Chen, P. Yang, S. Wang, and H. Duan, All-Dielectric Metasurfaces for Polarization Manipulation: Principles and Emerging Applications, Nanophotonics 9, 3755 (2020).

[9] A. Arbabi, Y. Horie, M. Bagheri, and A. Faraon, Dielectric Metasurfaces for Complete Control of Phase and Polarization with Subwavelength Spatial Resolution and High Transmission, Nat. Nanotechnol. 10, 937 (2015).

[10] R. C. Devlin, A. Ambrosio, N. A. Rubin, J. P. B. Mueller, and F. Capasso, Arbitrary Spin-to-Orbital Angular Momentum Conversion of Light, Science 358, 896 (2017).

[11] M. Kim, A. M. H. Wong, and G. V. Eleftheriades, Optical Huygens' Metasurfaces with Independent Control of the Magnitude and Phase of the Local Reflection Coefficients, Phys. Rev. X 4, 041042 (2014).

[12] A. C. Overvig, S. Shrestha, S. C. Malek, M. Lu, A. Stein, C. Zheng, and N. Yu, Dielectric Metasurfaces for Complete and Independent Control of the Optical Amplitude and Phase, Light Sci. Appl. 8, 92 (2019).

[13] Y. Bao, J. Ni, and C-W. Qiu, A Minimalist Single-Layer Metasurface for Arbitrary and Full Control of Vector Vortex Beams, Adv. Mater. 32, 1905659 (2020).

[14] Z. L. Deng, M. Jin, X. Ye, S. Wang, T. shi, J. Deng, N. Mao, Y. Cao, B. O. Guan, A. Alu, G. Li, and X. Li, FullColor Complex-Amplitude Vectorial Holograms Based on Multi-Freedom Metasurfaces, Adv. Funct. Mater. 30, 1910610 (2020).

[15] D. G. Baranov, Y. Ziao, I. A. Nechepurenko, A. Krasnok, A. Alù, and M. A. Kats, Nanophotonic Engineering of Far-Field Thermal Emitters, Nat. Mater. 18, 920 (2019).

[16] J.-J. Greffet, R. Carminati, K. Joulain, J.-P. Mulet, S. Mainguy, and Y. Chen, Coherent Emission of Light by Thermal Sources, Nature (London) 416, 61 (2002).

[17] K. Joulain and A. Loizeau, Coherent Thermal Emission by Microstructured Waveguides, J. Quant. Spectrosc. Radiat. Transfer 104, 208 (2007).

[18] C. M. Cornelius and J. P. Dowling, Modification of Planck Blackbody Radiation by Photonic Band-Gap Structures, Phys. Rev. A 59, 4736 (1999).

[19] S.-Y. Lin, J. G. Fleming, E. Chow, J. Bur, K. K. Choi, and A. Goldberg, Enhancement and Suppression of Thermal Emission by a Three-Dimensional Photonic Crystal, Phys. Rev. B 62, R2243 (2000).
[20] C. Luo, A. Narayanaswamy, G. Chen, and J. D. Joannopoulos, Thermal Radiation from Photonic Crystals: A Direct Calculation, Phys. Rev. Lett. 93, 213905 (2004).

[21] S. Y. Lin, J. Moreno, and J. G. Fleming, Three-Dimensional Photonic-Crystal Emitter for Thermal Photovoltaic Power Generation, Appl. Phys. Lett. 83, 380 (2003).

[22] T. Inoue, M. De Zoysa, T. Asano, and S. Noda, Realization of Dynamic Thermal Emission Control, Nat. Mater. 13, 928 (2014).

[23] J. A. Schuller, T. Taubner, and M. L. Brongersma, Optical Antenna Thermal Emitters, Nat. Photonics 3, 658 (2009).

[24] T. Weng, P. Li, D. N. Chigrin, A. J. Giles, F. J. Bezares, and O. J. Glembocki,. J. D. Caldwell and T. Taubner, PhononPolaritonic Bowtie Nanoantennas: Controlling Infrared Thermal Radiation at the Nanoscale, ACS Photonics 4, 1753 (2017).

[25] G. Lu, C. R. Gubbin, J. R. Nolen, T. Folland, M. J. Tadjer, S. De Liberato, and J.D. Caldwell, Engineering the Spectral and Spatial Dispersion of Thermal Emission via Polariton-Phonon Strong Coupling, Nano Lett. 21, 1831 (2021).

[26] X. Liu, T. Tyler, T. Starr, A. F. Starr, N. M. Jokerst, and W. J. Padilla, Taming the Blackbody with Infrared Metamaterials as Selective Thermal Emitters, Phys. Rev. Lett. 107, 045901 (2011).

[27] K. Ikeda, H. T. Miyazaki, T. Kasaya, K. Yamamoto, Y. Inoue, K. Fujimura, T. Kanakugi, M. Okada, K. Hatade, and S. Kitagawa, Controlled Thermal Emission of Polarized Infrared Waves from Arrayed Plasmon Nanocavities, Appl. Phys. Lett. 92, 021117 (2008).

[28] J. A. Mason, S. Smith, and D. Wasserman, Strong Absorption and Selective Thermal Emission from a Midinfrared Metamaterial, Appl. Phys. Lett. 98, 241105 (2011).

[29] P. J. Hesketh, J. N. Zemel, and B. Gebhart, Polarized Spectral Emittance from Periodic Micromachined Surfaces. II. Doped Silicon: Angular Variation, Phys. Rev. B 37, 10803 (1988).

[30] I. Celanovic, D. Perreault, and J. Kassakian, ResonantCavity Enhanced Thermal Emission, Phys. Rev. B 72, 075127 (2005).

[31] N. Dahan, A. Niv, G. Biener, Y. Gorodetski, V. Kleiner, and E. Hasman, Enhanced Coherency of Thermal Emission: Beyond the Limitation Imposed by Delocalized Surface Waves, Phys. Rev. B 76, 045427 (2007).

[32] B. J. Lee, C. J. Fu, and Z. M. Zhang, Coherent Thermal Emission from One-Dimensional Photonic Crystals, Appl. Phys. Lett. 87, 071904 (2005).

[33] A. Battula and S. C. Chen, Monochromatic Polarized Coherent Emitter Enhanced by Surface Plasmons and a Cavity Resonance, Phys. Rev. B 74, 245407 (2006).

[34] G. Biener, N. Dahan, A. Niv, V. Kleiner, and E. Hasman, Highly Coherent Thermal Emission Obtained by Plasmonic Bandgap Structures, Appl. Phys. Lett. 92, 081913 (2008).

[35] S. L. Wadsworth, P. G. Clem, E. D. Branson, and G. D. Boreman, Broadband Circularly-Polarized Infrared Emission from Multilayer Metamaterials, Opt. Mater. Express 1, 466 (2011).

[36] J. C. W. Lee and C. T. Chan, Circularly Polarized Thermal Radiation from Layer-by-Layer Photonic Crystal Structures, Appl. Phys. Lett. 90, 051912 (2007). 
[37] N. Dahan, Y. Gorodetski, K. Frischwasser, V. Kleiner, and E. Hasman, Geometric Doppler Effect: Spin-Split Dispersion of Thermal Radiation, Phys. Rev. Lett. 105, 136402 (2010).

[38] K. Frischwasser, I. Yulevich, V. Kleiner, and E. Hasman, Rashba-like Spin Degeneracy Breaking in Coupled Thermal Antenna Lattices, Opt. Express 19, 23475 (2011).

[39] L. Ouyang, W. Wang, D. Rosenmann, D. A. Czaplweski, J. Gao, and X. Yang, Near-Infrared Chiral Plasmonic Metasurface Absorbers, Opt. Express 26, 31484 (2018).

[40] S. A. Dyakov, V. A. Semenenko, N. A. Gippius, and S. G. Tikhodeev, Magnetic Field Free Circularly Polarized Thermal Emission from a Chiral Metasurface, Phys. Rev. B 98, 235416 (2018).

[41] S. Inampudi, J. Cheng, M. M. Salary, and H. Mosallaei, Unidirectional Thermal Radiation from a SiC Metasurface, J. Opt. Soc. Am. B 35, 39 (2018).

[42] H. Chalabi, A. Alù, and M. L. Brongersma, Focused Thermal Emission from a Nanostructured SiC Surface, Phys. Rev. B 94, 094307 (2016).

[43] M. Zhou, E. Khoram, D. Liu, B. Liu, S. Fan, M. L. Povinelli, and Z. Yu, Self-Focused Thermal Emission and Holography Realized by Mesoscopic Thermal Emitters, ACS Photonics 8, 497 (2021).

[44] G. Lozano, S. R. K. Rodriguez, M. A. Verschuuren, and J. G. Rivas, Metallic Nanostructures for Efficient LED Lighting, Light Sci. Appl. 5, e16080 (2016).

[45] A. Vaskin, R. Kolkowski, A. F. Koenderink, and I. Staude, Light-Emitting Metasurfaces, Nanophotonics 8, 1151 (2019).

[46] P. Wurfel and W. Ruppel, The Chemical Potential of Luminescent Radiation, J. Lumin. 24, 925 (1981).

[47] A. A. Maksimov, I. I. Tartakovskii, E. V. Filatov, S. V. Lobanov, N. A. Gippius, S. G. Tikhodeev, C. Schneider, M. Kamp, S. Maier, S. Hofling, and V. D. Kulakovskii, Circularly Polarized Light Emission from Chiral SpatiallyStructured Planar Semiconductor Microcavities, Phys. Rev. B 89, 045316 (2014).

[48] P. P. Iyer, R. A. DeCrescent, Y. Mohtashami, G. Lheureux, N. A. Butakov, A. Alhassan, C. Weisbuch, S. Nakamura, S. P. DenBaars, and J. A. Schuller, Unidirectional Luminescence from InGaN/GaN Quantum-Well Metasurfaces, Nat. Photonics 14, 543 (2020).

[49] M. Cotrufo, C. I. Osorio, and A. F. Koenderink, Spin-Dependent Emission from Arrays of Planar Chiral Nanoantennas due to Lattice and Localized Plasmonic Resonances, ACS Nano 10, 3389 (2016).

[50] A. Mohtashami, C. I. Osorio, and A. F. Koenderink, AngleResolved Polarimetry of Antenna-Mediated Fluorescence, Phys. Rev. Applied 4, 054014 (2015).

[51] D. Schanne, S. Suffit, P. Filloux, E. Lhuillier, and A. Degiron, Spontaneous Emission of Vector Vortex Beams, Phys. Rev. Applied 14, 064077 (2020).

[52] S. Liu et al. Light-Emitting Metasurfaces: Simultaneous Control of Spontaneous Emission and Far-Field Radiation, Nano Lett. 18, 6906 (2018).

[53] A. C. Overvig, S. C. Malek, and N. Yu, Multifunctional Nonlocal Metasurfaces, Phys. Rev. Lett. 125, 017402 (2020).
[54] A. C. Overvig, S. C. Malek, M. J. Carter, S. Shrestha, and N. $\mathrm{Yu}$, Selection Rules for Quasibound States in the Continuum, Phys. Rev. B 102, 035434 (2020).

[55] S. C. Malek, A. C. Overvig, A. Alù, and N. Yu, Resonant Wavefront-Shaping Flat Optics, arXiv:2009.07054.

[56] A. C. Overvig, N. Yu, and A. Alù, Chiral Quasi-Bound States in the Continuum, Phys. Rev. Lett. 126, 073001 (2021).

[57] A.C. Overvig and A. Alù, Wavefront-Selective Fano Resonant Metasurfaces, Adv. Photonics 3, 026002 (2021).

[58] K. Koshelev, S. Lepeshov, M. Liue, A. Bogdanov, and Y. Kivshar, Asymmetric Metasurfaces with High-Q Resonances Governed by Bound States in the Continuum, Phys. Rev. Lett. 121, 193903 (2018).

[59] D. Gabor, A New Microscopic Principle, Nature (London) 161, 777 (1948).

[60] D. A. B. Miller, L. Zhu, and S. Fan, Universal Modal Radiation Laws for All Thermal Emitters, Proc. Natl. Acad. Sci. U.S.A. 114, 4336 (2017).

[61] A. J. Kenyon, Erbium in Silicon, Semicond. Sci. Technol. 20, R65 (2005).

[62] A. C. Overvig, S. Shrestha, and N. Yu, Dimerized High Contrast Gratings, Nanophotonics 7, 1157 (2018).

[63] J. R. Piper and S. Fan, Total Absorption in a Graphene Monolayer in the Optical Regime by Critical Coupling with a Photonic Crystal Guided Resonance, ACS Photonics 1, 347 (2014).

[64] H. A. Haus, Waves and Fields in Optoelectronics (PrenticeHall, Englewood Cliffs, NJ, 1984).

[65] S. Fan, W. Suh, and J. D. Joannopoulos, Temporal CoupledMode Theory for the Fano Resonance in Optical Resonators, J. Opt. Soc. Am. A 20, 569 (2003).

[66] W. Suh, Z. Wang, and S. Fan, Temporal Coupled-Mode Theory and the Presence of Non-orthogonal Modes in Lossless Multimode Cavities, IEEE J. Quantum Electron. 40, 1511 (2004).

[67] R. Carminati and J.-J. Greffet, Near-Field Effects in Spatial Coherence of Thermal Sources, Phys. Rev. Lett. 82, 1660 (1999).

[68] K. Joulain, J.-P. Mulet, F. Marquier, R. Carminati, and J.-J. Greffet, Surface Electromagnetic Waves Thermally Excited: Radiative Heat Transfer, Coherence Properties and Casimir Forces Revisited in the Near Field, Surf. Sci. Rep. 57, 59 (2005).

[69] W. T. Lau, J.-T. Shen, G. Veronis, and S. Fan, Spatial Coherence of the Thermal Electromagnetic Field in the Vicinity of a Dielectric Slab, Phys. Rev. E 76, 016601 (2007).

[70] H. S. Nguyen, F. Dubois, T. Deschamps, S. Cueff, A. Pardon, J.-L. Leclercq, C. Seassal, X. Letartre, and P. Viktorovitch, Symmetry Breaking in Photonic Crystals: On-Demand Dispersion from Flatband to Dirac Cones, Phys. Rev. Lett. 120, 066102 (2018).

[71] J. R. Piper, V. Liu, and S. Fan, Total Absorption by Degenerate Critical Coupling, Appl. Phys. Lett. 104, 251110 (2014).

[72] Y. Jia, M. Qiu, H. Wu, Y. Cui, S. Fan, and Z. Ruan, Theory of Half-Space Light Absorption Enhancement for Leaky Mode Resonant Nanowires, Nano Lett. 15, 5513 (2015). 
[73] H. Cai, S. Srinivasan, D. A. Czaplewski, A. B. F. Martinson, D. J. Goszsola, L. Stan, T. Loeffler, S. K. R. S. Sankaranarayanan, and D. Lopez, Inverse Design of Metasurfaces with Non-local Interactions, npj Comput. Mater. 6, 116 (2020).

[74] R. F. Harrington, Time-Harmonic Electromagnetic Fields (Wiley-IEEE, New York, 2001).

[75] Y. Wang, A. C. Overvig, S. Shrestha, R. Zhang, R. Wang, N. $\mathrm{Yu}$, and L. Dal Negro, Tunability of Indium Tin Oxide Materials for Mid-infrared Plasmonics Applications, Opt. Mater. Express 7, 2727 (2017).

[76] S. M. Sze, Physics of Semiconductor Devices, 2nd ed. (Wiley-Interscience New York, 1981).

[77] R. A. Soref and B. R. Bennett, Electrooptical Effects in Silicon, IEEE J. Quantum of Electron. 23, 123 (1987).
[78] S. M. Rytov, Y. A. Kravtsov, and V. I. Tatarskii, Principles of Statistical Radiophysics 3 (Springer-Verlag, Berlin, 1989).

[79] R. E. Collin, Field Theory of Guided Waves, 2nd ed. (Wiley-IEEE, New York, 1990).

[80] Y. Zhou, I. I. Kravchenko, H. Wang, J. R. Nolen, G. Gu, and J. Valentine, Multilayer Noninteracting Dielectric Metasurfaces for Multiwavelength Metaoptics, Nano Lett. 18, 7529 (2018).

[81] Y. Zhao, M. A. Belkin, and A. Alù, Twisted Optical Metamaterials for Planarized Ultrathin Broadband Circular Polarizers, Nat. Commun. 3, 870 (2012).

[82] K. Tanaka, D. Arslan, S. Fasold, M. Steinert, J. Sautter, M. Falkner, T. Pertsch, M. Decker, and I. Staude, Chiral Bilayer All-Dielectric Metasurfaces, ACS Nano 14, 15926 (2020).

[83] K. Sakoda, Optical Properties of Photonic Crystals (Springer, Berlin, 2005). 